\title{
Singularity solution of Lanir's osmoelasticity: verification of discontinuity simulations in soft tissues
}

\author{
J. M. Huyghe · F. Kraaijeveld
}

Received: 16 June 2010 / Accepted: 28 November 2010 / Published online: 17 December 2010

(C) The Author(s) 2010. This article is published with open access at Springerlink.com

\begin{abstract}
The literature characterizes cartilaginous tissues as osmoviscoelastic. Understanding the damage and failure of these tissues is essential for designing treatments. To determine tissue strength and local stresses, experimental studies-both clinical and animal-are generally supported by computational studies. Verification methods for computational studies of ionized porous media including cracks are hardly available. This study provides a method for verification and shows its performance. For this purpose, shear loading of a finite crack is addressed analytically and through a commercial finite element code. Impulsive shear loading by two-edge dislocation of a crack was considered in a $2 \mathrm{D}$ plane strain model for an ionized porous medium. To derive the analytical solution, the system of equation is decoupled by stress functions. The shear stress distribution at the plane of the crack is derived using Fourier and Laplace transformations. The analytical solution for the shear stress distribution is compared with computer simulations in ABAQUS version 6.4-5. Decoupling of the equations makes it possible to solve some boundary value problems in porous media taking chemical effects into account. The numerical calculations underestimate the shear stress at the crack-tips. Mesh refinement increases accuracy, but is still low in the neighborhood of the crack-tips.
\end{abstract}

Keywords Dislocation - Donnan osmosis - Cartilaginous tissues - Shale · Porous media - Analytical solution .

Stress functions

J. M. Huyghe $(\varangle) \cdot$ F. Kraaijeveld

Department of Biomedical Engineering,

Eindhoven University of Technology,

Eindhoven, The Netherlands

e-mail: j.m.r.huyghe@tue.nl; jacques@wfw.wtb.tue.nl

\section{Introduction}

Many diseases are associated with localized mechanical failure and damage of tissues. Examples are cartilage damage (Wilson et al. 2004) and intervertebral disk herniation (Wognum et al. 2006). To determine tissue strength and local stresses, experimental studies (both clinical and animal) are supported by computational studies. Numerical methods have been developed to describe crack propagation in porous media (Armero and Callari 1999; Schrefler et al. 2004; Rethore et al. 2007). Usually they are not verified by analytical solutions. The verification is often restricted to a qualitative comparison with experimental measurements. However, two uncertainties are involved in this comparison. One is whether the numerical model actually solves the equation it claims to solve. The other is whether the equation describes the physical reality of the experiment. In order to test separately the former question, an analytical solution is a must. One important property of tissues is their swelling capacity. The presence of fixed charges in the tissues causes differences in ion concentrations with the surrounding fluid and therefore a Donnanosmotic pressure (Maroudas et al. 1991; Urban and Roberts 2003). Subsequently, the deformation of tissues depends not only on mechanical factors, but also on biochemical factors.

Osmotically swelling porous media have been modeled by many groups. Lanir (1987) extended the Biot model (Biot 1941) for incompressible constituents, namely the solid matrix and interstitial fluid, by including Donnanosmotic pressure, but neglecting the influence of ion flow. Lai et al. (1991) extended Lanir's model to the triphasic theory including ionic diffusion-convection. Huyghe and Janssen (1999) developed an electrochemomechanical model using four constituents. It has been implemented for finite 
deformations and verified for one-dimensional pressure tests by Frijns et al. (1997). Gu et al. (1998) developed a multiionic model. van Loon et al. (2003) implemented a 3D Finite Element model of the quadriphasic model while Iatridis et al. developed a closely related PEACE-model of the intervertebral disk (Iatridis et al. 2003). Chen et al. (2006) simulate the curling behavior of cartilage using similar finite element modeling of finite deformation mechano-electro-chemical coupling. Meerveld et al. (2003) validated the quadriphasic model by analytical solutions. The confined and unconfined compression tests are common validation tools. Wilson et al. (2005a) compared the biphasic swelling model (Lanir 1987) with the quadriphasic model (Huyghe and Janssen 1997) and showed that the flow of ions indeed influences the pathway to equilibrium with the external fluid. Although the mechano-electro-chemical models are more realistic in transient behavior, Lanir's model is a reliable approximation for the modeling of ionized porous media, such as the intervertebral disk (Schroeder et al. 2006). The last method is less complex and computationally less expensive than the former.

Some methods for validation have already been indicated above; however, previous research has been restricted to continuous media. There are hardly any methods for validation of computational efforts of fractured porous media. Tests used in solid mechanics, like the three-point bending test or analytical solutions from linear fracture mechanics, are not suitable for porous media since the influence of fluid flow is not accounted for. Crack-tip analysis and localization in Atkinson and Craster (1991), Loret and Radi (2001), and Larsson and Larsson (2000) have been topics of interest, but only give local trends. Rice and Cleary developed an analytical solution for earthquake (shear faulting) predictions (Rice and Cleary 1976), which has shown to predict aftershocks. Pure shearing mode of a crack is considered, and special treatment of the crack is circumvented. Rice and Cleary's method uses Biot functions (Biot 1956), which are similar to the Airy functions in solid linear fracture mechanics. However, these functions can only be applied when there is compressibility in the model. Booker (1974) calculated the dimensionless stress field along a shear fault for a fully saturated incompressible porous medium by decoupling the equations with stress functions according McNamee and Gibson (1960) (Verruijt 1971; Detournay and Cheng 1993). Rice and Cleary (1976) showed that both methods find the same result in the limiting case of incompressibility. The objective of this study is to establish a 2D method to study the performance of computational modeling of ionized porous media with cracks, namely an analytical solution suited for comparison with numerical calculations. In particular, the method by Booker (Booker 1974; Nur and Booker 1972) is generalized to ionized porous media.

\section{Method}

In this section, the model by Lanir (1987) is stated shortly. A perturbation on a homogeneous situation is considered; therefore, the constitutive equations are linearized. The case of interest is given, after which the analytical solution is derived and numerical calculations are presented.

\subsection{Governing equations}

Osmoelastic media have large negatively charged groups attached to the solid matrix. Counter charges are present in the fluid making the medium electrically neutral. Due to the fixed charges, the total ion concentration inside the medium is higher than in the surrounding fluid. This excess of ion particles leads to an osmotic pressure difference, which causes swelling of the medium. Lanir's osmoelastic model (Lanir 1987) assumes that small ions are always in equilibrium with the external salt concentration. This means that ion contribution is neglected and the medium is described by two constituents only: the solid (s) and the fluid (f). The constituents are assumed to be incompressible. Infinitesimal deformations are assumed. The material is linear elastic, isothermal, isotropic, homogeneous and fully saturated. Osmotic swelling is included by the introduction of the chemical potential of the fluid, which is a measure for the free energy of the fluid. The chemical potential of the fluid $\mu^{\mathrm{f}}$ is defined per unit volume fluid and will be denoted by chemical potential:

$\mu^{\mathrm{f}}=p-\pi$.

with $\pi$ the osmotic pressure and $p$ the hydrostatic pressure. The osmotic pressure is determined by the empirical Van ' $t$ Hoff equation. This relation defines the osmotic pressure in terms of concentrations of free cations $c^{+}$, anions $c^{-}$, osmotic coefficient $\Gamma$, gas constant $R$ and temperature $T$.

$\pi=R T \Gamma\left(c^{+}+c^{-}\right), \quad c^{+}+c^{-}=\sqrt{\left(c^{f c}\right)^{2}+4\left(c^{e x}\right)^{2}}$

This osmotic pressure holds outside as well as inside the medium, but outside the medium the fixed charge density $c^{f c}$ is zero and the osmotic coefficient may be different. Electroneutrality holds, therefore, the amount of negative charges are equal to the amount of positive charges: $c^{-}+c^{f c}=c^{+}$. Furthermore, the seepage flux q follows Darcy's law in presence of concentration gradients. The total equations are given by (3).

$$
\begin{array}{ll}
\text { Momentum } & \nabla \cdot \sigma=\nabla \cdot \sigma_{\mathrm{e}}-\nabla\left(\mu^{\mathrm{f}}+\pi\right)=\mathbf{0} \\
\text { Stress-strain } & \sigma_{\mathrm{e}}=2 \mu \epsilon+\lambda \operatorname{tr}(\epsilon) \boldsymbol{I} \\
\text { Mass } & \nabla \cdot \frac{\partial \mathbf{u}}{\partial t}+\nabla \cdot \mathbf{q}=0 \\
\text { Darcy's law } & \mathbf{q}=-\boldsymbol{K} \cdot \nabla \mu^{\mathrm{f}} \\
\text { Swelling } \quad \pi=R T \Gamma \sqrt{\left(c^{\mathrm{fc}}\right)^{2}+4\left(c^{\mathrm{ex}}\right)^{2}} \\
\text { Fixed charge } c^{\mathrm{fc}}=\frac{\phi_{i}^{\mathrm{f}} c_{0}^{\mathrm{fc}}}{\operatorname{tr}(\epsilon)+\phi_{i}^{\mathrm{f}}}
\end{array}
$$


The parameters $\mu$ and $\lambda=\kappa-2 \mu / 3$ are the Lamé constants. $\nu, \kappa$ and $\mu$ are the Poisson's ratio, bulk modulus and shear modulus, respectively. The tensor $\boldsymbol{K}=K \boldsymbol{I}$ is the hydraulic permeability tensor and is assumed isotropic and constant in time and space.

The presence of ions fixed to the solid matrix results in prestress of the solid matrix at initial condition. $\epsilon$ is the strain tensor relative to the stress-free configuration, which includes the initial strain $\epsilon_{i}$ and the deformation $\epsilon-\epsilon_{i}=$ $1 / 2\left\{\nabla \mathbf{u}+(\nabla \mathbf{u})^{T}\right\}$ relative to the initial state. Similarly, the fixed charge density is calculated from the fixed charge density at stress-free configuration $c_{0}^{\mathrm{fc}}$ and initial volume fraction $\phi_{i}^{f}$. Under assumption of small strains, the swelling equation is linearized around the initial strain.

$$
\begin{aligned}
\pi= & \left.\pi\right|_{\epsilon_{i}}+\frac{\partial \pi}{\partial \operatorname{tr}(\epsilon)} \mid \epsilon_{i}\left(\operatorname{tr}(\epsilon)-\operatorname{tr}\left(\epsilon_{i}\right)\right) \\
= & \left.\pi\right|_{i}+\frac{\partial \pi}{\partial c^{\mathrm{fc}}} \frac{\partial c^{\mathrm{fc}}}{\partial \operatorname{tr}(\epsilon)} \mid \epsilon_{i}\left(\operatorname{tr}(\epsilon)-\operatorname{tr}\left(\epsilon_{i}\right)\right) \\
= & R T \Gamma \sqrt{\left(c_{i}^{\mathrm{fc}}\right)^{2}+4\left(c^{e x}\right)^{2}} \\
& -R T \Gamma \frac{c_{i}^{\mathrm{fc}}}{\sqrt{\left(c_{i}^{\mathrm{fc}}\right)^{2}+4\left(c^{e x}\right)^{2}}} \frac{c_{i}^{\mathrm{fc}}}{\left(\operatorname{tr}\left(\epsilon_{i}\right)+\phi_{i}^{f}\right)}\left(\operatorname{tr}(\epsilon)-\operatorname{tr}\left(\epsilon_{i}\right)\right)
\end{aligned}
$$

with $c_{i}^{\text {fc }}$ the initial fixed charge density and is used to compute the fixed charge density at stress-free state. For simplification we introduce the constants $C_{0}=\sqrt{\left(c_{i}^{\mathrm{fc}}\right)^{2}+4\left(c^{\mathrm{ex}}\right)^{2}}$, the initial concentrations, and $C_{1}=\frac{\left(c_{i}^{\mathrm{fc}}\right)^{2}}{\left(\phi_{0}^{\mathrm{f}}+t r\left(\epsilon_{i}\right)\right) C_{0}}$, the change in concentrations due to a unit volumetric strain. Then, the osmotic pressure in terms of deformation becomes

$\pi(\epsilon)=R T \Gamma\left(C_{0}-\operatorname{tr}\left(\epsilon-\epsilon_{i}\right) C_{1}\right)$.

Subsequently, system (3) can be rewritten to

$2 \mu \nabla^{2} \mathbf{u}+(c-2 \mu) \nabla \operatorname{tr}\left(\epsilon-\epsilon_{i}\right)-\nabla \mu^{\mathrm{f}}=0$

$\frac{\partial \operatorname{tr}\left(\epsilon-\epsilon_{i}\right)}{\partial t}-\nabla \cdot\left(K \nabla \mu^{\mathrm{f}}\right)=0$,

with $c=2 \mu+\lambda+R T \Gamma C_{1}, \mu^{\mathrm{f}}=\mu^{\mathrm{f}}+R T \Gamma C_{0}$.

\subsection{Singularity case}

A rectangular piece of material is considered with a crack in the middle in the $x, z$-plane of length $2 L$. The crack is closed. Fluid flow across the crack is allowed, by equating the chemical potential at either side of the crack. Shear faulting is impulsively induced as a small displacement enforced on the upper and lower crack surface in opposite direction. This is shown in Fig. 1. The result is a steep increase in shear stress and fluid flow.

The upper crack surface is positioned at $z=0^{+}$and the lower crack surface at $z=0^{-}$. If we define $\mathbf{u}=(u, w)^{T}$ as

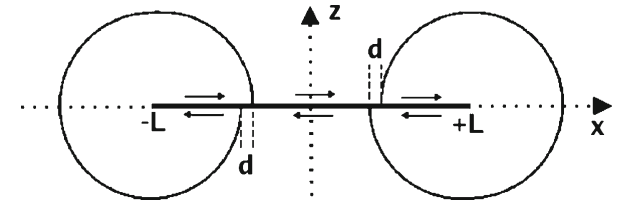

Fig. 1 Two-edge dislocations of magnitude $d$ over crack length $2 L$

the displacement field, we can define the jump over the crack surface $[u]$ as

$[u](x, t)=u\left(x, 0^{+}, t\right)-u\left(x, 0^{-}, t\right)$.

Then the perturbation can mathematically be defined as

$$
\begin{aligned}
& {[u]=f(x) \mathcal{H}(t)} \\
& f(x)=d(\mathcal{H}(x+L)-\mathcal{H}(x-L))= \begin{cases}d & \text { if } x \in[-L, L] \\
0 & \text { otherwise }\end{cases}
\end{aligned}
$$

Here, $d$ denotes the magnitude of the dislocation and therefore the magnitude of the jump in shear direction. $f(x)$ is called the slip function, $\mathcal{H}$ is the Heaviside function. Since the crack is closed there is no jump in displacement in $z$-direction $([w]=0)$. Furthermore, we have continuity of stresses $\left(\left[\sigma_{z z}\right]=0\right)$.

We are interested in the shear stress at the crack surfaces, analytically and numerically.

\section{Analytical solution}

The model (6) is strongly coupled. For the derivation of the analytical solution, decoupling of the total system of equations is needed. Following Booker (1974), the solution is found using integral transformations of Laplace and Fourier.

\subsection{Decoupling}

The system (6) is decoupled by the introduction of stress functions (McNamee and Gibson 1960). The stress function $S(x, z, t)$ plays a role in the momentum equations and $E(x, z, t)$ a larger role in the mass equation (Appendix).

The stress function $S(x, z, t)$ is harmonic, meaning $\nabla^{2} S=0$ holds. The momentum equation is automatically satisfied, if for the chemical potential the next holds:

$\mu^{\mathrm{f}}=\operatorname{ctr}(\epsilon)-2 \mu \frac{\partial S}{\partial z}$.

The second stress function $E(x, z, t)$ is introduced such that

$\operatorname{tr}\left(\epsilon-\epsilon_{i}\right)=\nabla^{2} E$. 
Substituting Eqs. (9) and (10) in Eq. (6) decouples the system of equations (McNamee and Gibson 1960):

$\nabla^{2} S=0$,

$\nabla^{2} \frac{\partial E}{\partial t}-c K \nabla^{4} E=0$

The number of equations has not been reduced, but the total system of equations, Eq. (11), has been rewritten to decoupled partial differential equations, which are easier to solve. A complete overview is found in "Appendix".

\subsection{Governing equations in Laplace-Fourier domain}

Any function can be split up into an even and an odd portion. McNamee and Gibson (1960) only consider symmetric loading and therefore only use the symmetric part of Fourier transforms. To be able to consider more general cases, general Fourier and Laplace transformations are used (Booker 1974). Fourier transforms carry a tilde and depend on variables $k, z$ and $t$. Laplace-Fourier transforms carry a hat and depend on variables $k, z$ and $s$. Since a shear crack in the $x, z$ plane is considered, the displacement jump will be over $z=0$ in the $x$ direction, Fourier transformation along the crack is performed, thus over the $x$ direction (Appendix). The forward Fourier transformation in $x$ becomes

$\tilde{E}(k, z, t)=\int_{-\infty}^{\infty} E(x, z, t) \mathrm{e}^{-i k x} \mathrm{~d} x$.

Equivalently, the Laplace transformation is given by

$\hat{E}(k, z, s)=\int_{0}^{\infty} \tilde{E}(k, z, t) \mathrm{e}^{-s t} \mathrm{~d} t$.

The result is a second-order ordinary differential equation (ODE) for Laplace-Fourier transform $\hat{S}$ of $S$ and a fourth-order ODE for Laplace-Fourier transform $\hat{E}$ of $E$. The dependent and independent variables are written in terms of the stress functions. From this, boundary conditions in the form of jump conditions are derived. In total, the two boundary value problems (14) and (15) are found.

$$
\begin{aligned}
& \left(\frac{\partial^{2}}{\partial z^{2}}-k^{2}\right) \hat{S}=0 \\
& {[\hat{S}]=0 \quad\left[\frac{\partial \hat{S}}{\partial z}\right]=\frac{i k}{s} \tilde{f}(k) .}
\end{aligned}
$$

and

$$
\begin{aligned}
& \left(\frac{\partial^{2}}{\partial z^{2}}-k^{2}\right)\left(c K\left(\frac{\partial^{2}}{\partial z^{2}}-k^{2}\right)-s\right) \hat{E}=0 . \\
& {[\hat{E}]=\frac{-i}{k s} \tilde{f}(k) \quad\left[\frac{\partial \hat{E}}{\partial z}\right]=0,}
\end{aligned}
$$

$\left[\frac{\partial^{2} \hat{E}}{\partial z^{2}}\right]=-\frac{i s}{c p} \tilde{f}(s)(c-2 \mu) \quad\left[\frac{\partial^{3} \hat{E}}{\partial z^{3}}\right]=0$.

with $\tilde{f}(k)$ the Fourier transform of slip function $f(x)$ :

$\tilde{f}(k)=\int_{-L}^{L} \mathrm{de}^{-i k x} \mathrm{~d} x=\frac{2 \mathrm{~d} \sin (L k)}{k}$.

\subsection{Solution}

Previously the boundary value problem (BVP) in the Laplace-Fourier domain has been derived. This BVP is solved by splitting of the domain. The resulting solution is transformed from the Laplace domain to normal time domain. Since the interest lies in the shear stress around the crack-tip, the equations are simplified by taking $z=0$. Then, back transformation from the Fourier domain to the normal $x, z$ domain is performed.

The inverse transform becomes (17).

$E(x, z, t)=\frac{1}{2 \pi} \int_{-\infty}^{\infty} \tilde{E}(k, z, t) \mathrm{e}^{+i k x} \mathrm{~d} k$

The inverse Laplace transformation is performed in "Appendix" using Abramowitz and Stegun (1970).

\subsubsection{Solution in Laplace-Fourier domain}

The solutions in Laplace-Fourier domain are derived by splitting the domain in an upper plane $\left(z>0: \hat{S}_{+}, \hat{E}_{+}\right)$and lower plane $\left(z<0: \hat{S}_{-}, \hat{E}_{-}\right)$. Far from the crack the solution is not affected by the jump, so the solution has to approach zero for $z \rightarrow \pm \infty$.

For stress function $\hat{S}$, the general solution in the upper plane is given by $\hat{S}_{+}=\gamma_{+} \mathrm{e}^{-|k| z}$ and for lower plane $\hat{S}_{-}=$ $\gamma_{-} \mathrm{e}^{+|k| z}$. Then, the jump condition reduces to

$$
\begin{aligned}
{[\hat{S}] } & =\lim _{z \downarrow 0} \hat{S}-\lim _{z \uparrow 0} \hat{S} \\
& =\hat{S}_{+}(k, 0, s)-\hat{S}_{-}(k, 0, s)=\gamma_{+}-\gamma_{-} .
\end{aligned}
$$

Through linear algebra, one derives that the solution of (14) is given by

$\hat{S}(k, z, s)=\frac{-i \operatorname{sign}(k)}{2 s} \tilde{f}(k) \mathrm{e}^{-|k||z|}$.

with $\operatorname{sign}(k)=\frac{k}{|k|}$. A similar solution of the mass Eq. (15) is now determined and is given by

$$
\begin{aligned}
\hat{E}(k, z, s)= & -\operatorname{sign}(z)\left\{\left(\frac{i k \mu \tilde{f}(k) K}{s^{2}}+\frac{i \tilde{f}(k)}{2 k s}\right) \mathrm{e}^{-|k||z|}\right. \\
& \left.+\left(\frac{i k \mu \tilde{f}(k) K}{s^{2}}\right) \mathrm{e}^{-\frac{1}{c K} \sqrt{k^{2} c K+s}|z|}\right\},
\end{aligned}
$$




\subsection{Inverse transformation of the solution}

The derived solutions are still in the Laplace-Fourier domain. Back transformations have to be performed. The solutions involve several terms dependent on Laplace variable $s$. The stress function $\hat{S}$ contains only a factor $1 / s$, which becomes the Heaviside function by back transformation. So the inverse Laplace for stress function $\hat{S}$ is

$\tilde{S}(k, z, t)=-\frac{i \operatorname{sign}(k)}{2} \tilde{f}(k) \mathrm{e}^{-|k||z|} \mathcal{H}(t)$.

The inverse Laplace of stress function $\hat{E}$ is more difficult. It contains factors $1 / s, 1 / s^{2}$ but also a factor $\frac{\mathrm{e}^{-\frac{|z| \sqrt{k^{2} c K+s}}{\sqrt{c K}}}}{s^{2}}$. For deriving the inverse of the latter term we need to perform some mathematical manipulations, using the shift theorem and convolution theorem (Appendix 7). The result is

$$
\begin{aligned}
\tilde{E}(k, z, t)= & -\frac{i \mu \tilde{f}(k) \operatorname{sign}(z)}{2 c k}\left\{\left[\frac{c}{\mu}+|z||k|\right] \mathrm{e}^{-|k||z|} \mathcal{H}(t)\right. \\
& -c K\left(k^{2} t+\frac{|z||k|}{2 c K}\right) \mathrm{e}^{|k||z|} \operatorname{erf} c\left(\lambda^{+}\right) \\
& \left.+c K\left(k^{2} t-\frac{|z||k|}{2 c K}\right) \mathrm{e}^{-|k||z|} \operatorname{erf} c\left(\lambda^{-}\right)\right\} .
\end{aligned}
$$

with $\lambda^{ \pm}=|k| \sqrt{c K t} \pm|z| / \sqrt{4 c K t}$. The shear stress is then given by

$$
\begin{aligned}
\tilde{\sigma}_{x z}= & 2 \mu i k\left\{\frac{\partial \tilde{E}}{\partial z}-z \frac{\partial \tilde{S}}{\partial z}\right\} \\
= & \frac{-\mu^{2} \tilde{f}(k)|k|}{c}\left\{\left[-\left(1-\frac{c}{\mu}\right)(1-|z||k|)\right] \mathrm{e}^{-|k||z|} \mathcal{H}(t)\right. \\
& +c K\left(k^{2} t+\frac{|z||k|+1}{2 c K}\right) \mathrm{e}^{|k||z|} \operatorname{erf} c\left(\lambda^{+}\right) \\
& +c K\left(k^{2} t-\frac{|z||k|-1}{2 c K}\right) \mathrm{e}^{-|k||z|} \operatorname{erf} c\left(\lambda^{-}\right) \\
& \left.-2 k^{2} \frac{\sqrt{c K t}}{\sqrt{\pi}} \mathrm{e}^{-\left(k^{2} c K t+z^{2} /(4 c K t)\right)}\right\}
\end{aligned}
$$

\subsection{Shear stress}

The expression (23) simplifies to (24) for $z=0$, i.e. along the crack surface.

$$
\begin{aligned}
\left.\tilde{\sigma}_{x z}\right|_{z=0}= & \frac{-\mu^{2} \tilde{f}(k)|k|}{c}\left\{\left[-\left(1-\frac{c}{\mu}\right)\right] \mathcal{H}(t)\right. \\
& +\left(2 k^{2} c K t+1\right) \operatorname{erf} c(|k| \sqrt{c K t}) \\
& \left.-2 \frac{\sqrt{k^{2} c K t}}{\sqrt{\pi}} \mathrm{e}^{-\left(k^{2} c K t\right)}\right\}
\end{aligned}
$$

Back transformation from Fourier domain is performed using the repeated integrals of the complementary error function (Appendix). Numerical Fourier integration is not possible since the integrant does not go to zero for increasing Fourier variable $k$. The shear stress is given by Eq. (25)

$\sigma_{x z}(x, 0, t)=-2 \mu \frac{d \mathcal{H}(t)}{\pi\left(L^{2}-x^{2}\right)}\{L-\mu K F(x, t)\}$.

with

$$
\begin{aligned}
F(x, t)= & 2 t\left\{\frac{(L-x)}{(L+x)^{2}}\left(1-\mathrm{e}^{-\frac{(L+x)^{2}}{4 c K t}}\right)\right. \\
& \left.+\frac{(L+x)}{(L-x)^{2}}\left(1-\mathrm{e}^{-\frac{(L-x)^{2}}{4 C K t}}\right)\right\}
\end{aligned}
$$

The expansions (25) and (26) reduce to Eq. (16) of Booker if we replace expression $c=2 \mu+\lambda+R T C_{1}$ by $c=2 \mu+\lambda$, i.e. if we neglect osmotic effects. When $t \rightarrow \infty$, the function $F \rightarrow 0$ and the shear stress is at equilibrium. Using Taylor expansions, it is found that the shear stress at equilibrium is

$\sigma_{x z}\left(x, 0, t_{\text {equi }}\right)=-\frac{2 \mu d}{\pi\left(L^{2}-x^{2}\right)}\left(L-\frac{\mu K L}{c K}\right)$.

The equilibrium stress also indicates a high singularity near the crack-tips. As expected, the influence of the osmotic prestress is low on the shear stress at equilibrium, but is not negligible. Furthermore, the osmotic prestress influences the behavior in time.

\subsection{Chemical potential and flow}

To find the distribution for the chemical potential and the flow, it is possible to numerically integrate Eq. (9), but it is also possible to derive an analytical expression for the chemical potential. The chemical potential does not only satisfy Eq. (9), but it also satisfies:

$$
\left(\frac{\partial}{\partial t}-c K \nabla^{2}\right) \mu^{\mathrm{f}}=2 \mu \frac{\partial^{2} S}{\partial z \partial t} .
$$

This equation is found similar to Nur and Booker (1972) for hydraulic pressure by substituting (9) into (11b) and using that $S$ is harmonic. Since $S$ is time independent, the right side of Eq. (28) is zero. Equation (28) is transformed into polar coordinates about the crack tip. Assuming separation of variables, we write the solution as a product of a function depending on the radial coordinate $r$ and a function depending on the angle $\theta$ with the $x$-axis. The axisymmetric function is obtained using Hankel transformation (Booker 1974; Nur and Booker 1972) (Appendix). The result is 


$$
\begin{array}{r}
\mu^{\mathrm{f}}(x, z, t)=\frac{\mu \mathrm{d} z}{\pi}\left[\frac{1}{(x-L)^{2}+z^{2}}-\frac{1}{(x+L)^{2}+z^{2}}\right] \\
-\frac{\mu d z}{\pi} \mathrm{e}^{-z^{2} / 4 c K t}\left[\frac{\mathrm{e}^{-(x-L)^{2} / 4 c K t}}{(x-L)^{2}+z^{2}}-\frac{\mathrm{e}^{-(x+L)^{2} / 4 c K t}}{(x+L)^{2}+z^{2}}\right]
\end{array}
$$

From this, we can derive the flow over the crack (n vector normal to the crack, $z=0$ ):

$$
\begin{aligned}
\mathbf{q} \cdot \mathbf{n}(x, 0, t)= & -K \frac{\partial \mu^{\mathrm{f}}}{\partial z}(x, 0, t) \\
= & -K \frac{\mu d}{\pi}\left[\frac{1}{(x-L)^{2}}-\frac{1}{(x+L)^{2}}\right] \\
& +K \frac{\mu d}{\pi}\left[\frac{\mathrm{e}^{-(x-L)^{2} / 4 c K t}}{(x-L)^{2}}-\frac{\mathrm{e}^{-(x+L)^{2} / 4 c K t}}{(x+L)^{2}}\right]
\end{aligned}
$$

and the flow along the crack ( $\mathbf{t}$ vector in direction of crack-tip $x=+L)$ :

$\left.\mathbf{q} \cdot \mathbf{t}\right|_{z=0}=-K \frac{\partial \mu^{\mathrm{f}}}{\partial x}(x, 0, t)=0$

Equation (31) is not a prescribed boundary condition along the crack. It is the combined result of continuity of $\mu^{\mathrm{f}}$ across the crack and the symmetry inherent to the problem.

\section{Numerical example}

For simulations, Finite Element package ABAQUS 6.4-5 has been used. Lanir's model is implemented by Wilson et al. (2005b), but with a Neo-Hookean material law. This law reduces to the material as used here for small strains. Quadrilateral linear elements are used. The mesh is given in Figs. 2 and 3 . A crack is created bluntly by tying two meshes together except for $\mathrm{x}$-displacement across the crack and crack-tips. A contact condition is imposed between the crack surfaces. The crack-tips are not restricted in $x$-direction in order to have a pure symmetric reaction left and right from the crack-tip. This also implies that for boundary conditions a pure Heaviside function cannot be imposed, but the heaviside function is approximated by change over a length $\Delta x$, i.e. the length of the unconstraint interval around the crack-tip. This way a piece of medium of size $8 \mathrm{~mm} \times 6 \mathrm{~mm}$ with a fixed charge density of $c_{0}^{\mathrm{fc}}=0.2 \mathrm{mmol} / \mathrm{ml}$, a bulk modulus of $\kappa=0.5 \mathrm{MPa}$ and a shear modulus of $\mu=0.375 \mathrm{MPa}$ is created. These properties approximate the properties of disk tissue (Wognum et al. 2006; van Loon et al. 2003). The size is chosen sufficiently large that boundaries have no effect on the result using Eq. (29).

All boundaries are restricted in normal behavior, i.e. left side is restricted in the $x$-direction, etc,. The result is that the crack location and length does not change under osmotic

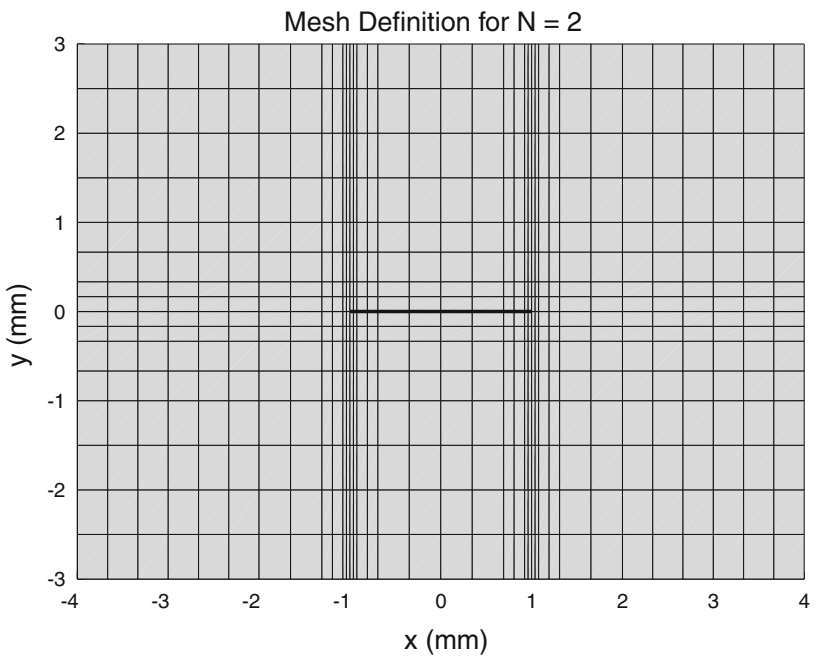

Fig. 2 Mesh creation for 576 elements. Mesh is refined toward the areas of interest: the crack-tips

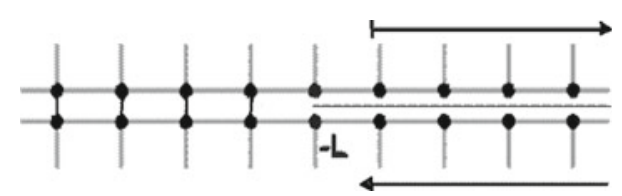

Fig. 3 Close up mesh at crack-tip $x=-L$. Nodes are tied outside the crack $(|x|>1)$. Contact is defined on the crack surfaces $x<|L|$, $z=0^{ \pm}$. The crack-tips $x= \pm L$ are free

loading. The medium is in equilibrium with an external salt concentration. This means that at all boundaries fluid flow is free and $\mu_{i n}^{\mathrm{f}}=\mu_{e x}^{\mathrm{f}}$ holds, i.e. the chemical potential of the fluid in the medium at the boundaries are equal to the external chemical potential. To prevent the crack from opening, the nodes at the crack surface are fixed in $z$-direction. This is in agreement with the jump condition $[w]=\left[u_{z}\right]=0$. After letting the medium reach equilibrium, an impulsive shear load is applied on the crack surfaces. A displacement smaller than the width of one element is imposed on the crack surfaces, namely a displacement of a magnitude of $d / 2$ for the upper crack surface in positive $x$-direction and lower surface in opposite direction.

Similar as in space, it is not possible to impose an impulsive loading in time, but it is approximated by changing the conditions over a time length $\Delta t$. The influence of time step is studied. After that, the calculations are run until steady state is reached. Shear stress in the nodes are calculated by averaging over the neighboring integration points. The change in shear stress is calculated by deducting the initial shear stress distribution from the state of interest. The effect of spatial discretization size is studied by considering three meshes from coarse to fine of mesh size 144 elements, 576 elements and 2304 elements. The parameters are given in Table 1. 
Table 1 Model parameters for numerical and analytical models

\begin{tabular}{llllllll}
\hline $\mathrm{R}$ & $=$ & 8.3145 & {$[\mathrm{~N} \mathrm{~mm} / \mathrm{mmol} \mathrm{K}]$} & $\mathrm{T}$ & $=$ & 298 & {$[\mathrm{~K}]$} \\
$\mu$ & $=$ & 0.375 & {$[\mathrm{MPa}]$} & $v$ & $=$ & 0.2 & {$[-]$} \\
$\kappa$ & $=$ & 0.5 & {$[\mathrm{MPa}]$} & & & & \\
$\phi_{i}^{\mathrm{f}}$ & $=$ & 0.8 & {$[-]$} & $K$ & $=$ & $0.28 \mathrm{e}-3$ & {$\left[\mathrm{~mm}^{4} / \mathrm{Ns}\right]$} \\
$c_{\mathrm{ex}}$ & $=$ & $0.15 \mathrm{e}-3$ & {$\left[\mathrm{mmol} / \mathrm{mm}^{3}\right]$} & $c_{i}^{\mathrm{fc}}$ & $=$ & $-0.2 \mathrm{e}-3$ & {$\left[\mathrm{mmoleq}^{3} \mathrm{~mm}^{3}\right]$} \\
$d$ & $=$ & 0.004 & {$[\mathrm{~mm}]$} & $L$ & $=$ & 1.0 & {$[\mathrm{~mm}]$} \\
\hline
\end{tabular}
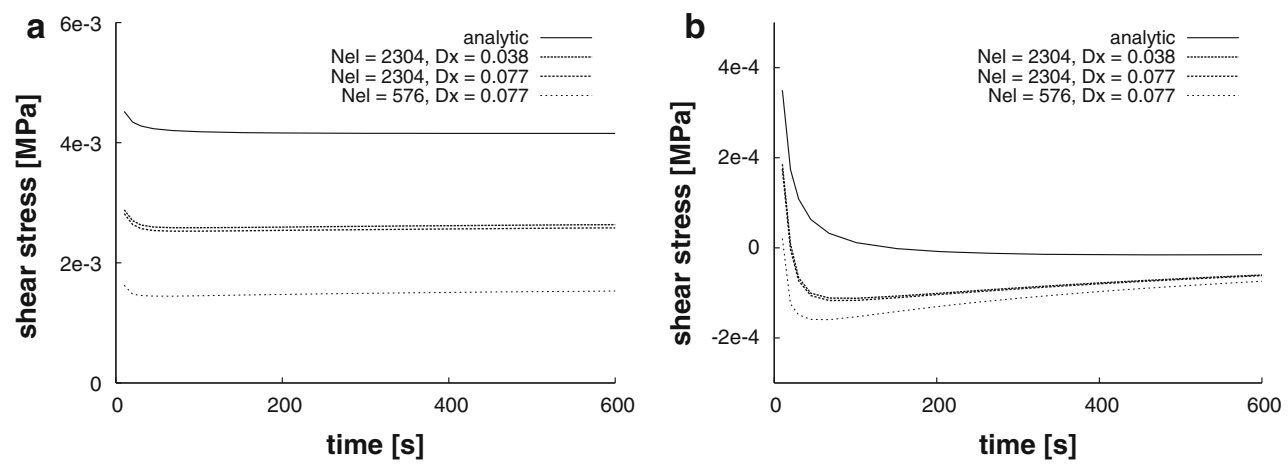

Fig. 4 Consolidation for different calculations at $x=1.0769 \mathrm{~mm}$. With refinement, the time-dependent behavior of the analytical solution is better approximated except for an offset. a Shows the data with the offset, $\mathbf{b}$ without the offset
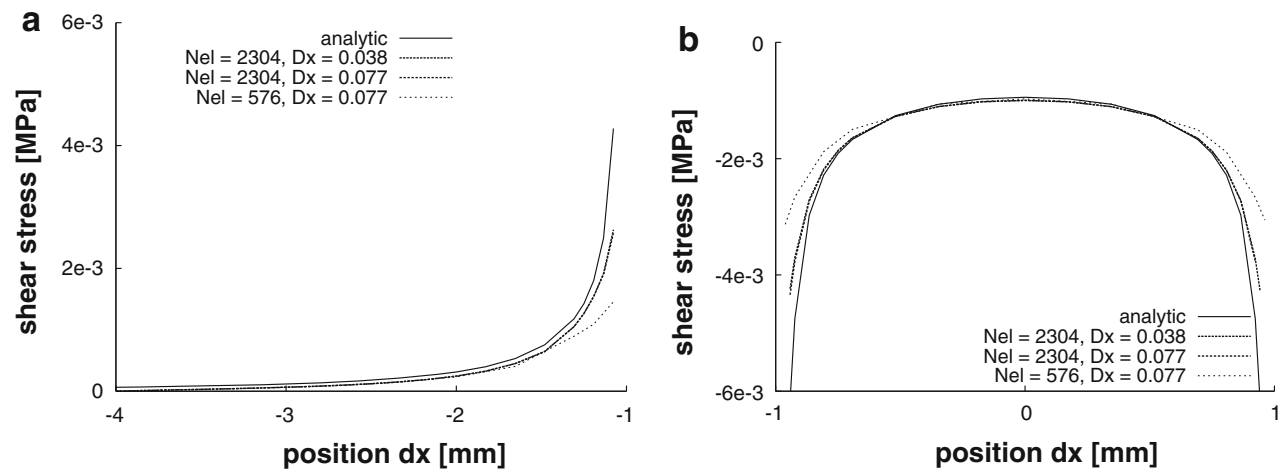

Fig. 5 Shear stress distribution at $t=30.0 \mathrm{~s}$. A high singularity is seen in all cases. The stresses left (a) and right (b) from the crack-tip, are equal in magnitude. The numerical shear stress converges to the analytical shear stress upon refinement, except in vicinity of the crack-tips $x= \pm-1$

\section{Results}

As the result of impulsive loading, the medium reacts as if incompressible, leading to high shear stress. Then, the fluid flow starts and relaxation takes place. Consolidation takes place. This is seen in the Eq. (25), but also Fig. 4.

The dislocation causes compression above the crack and expansion underneath the crack for $x>0$ and viceversa for $x<0$. The normal stresses are antisymmetric with respect to the $z$-axis. The shear stress is symmetric with respect to the $z$-axis and antisymmetric with respect to the crack-tips (Fig. 5). The values left and right of the crack-tip are equal in magnitude (not sign), also for the numerical models. In the crack-tips, the shear stress undergoes a high singularity $(x= \pm 1)$.
The dislocation causes for $x>0$ compression above the crack and expansion underneath the crack. The normal stresses and the chemical potential are antisymmetric about the $z$-axis and $x$-axis. In Fig. $6 \mathrm{a}$ and $\mathrm{b}$, it is seen that at the crack-tips the compression produces a positive chemical potential and extension produces a negative chemical potential. Fluid flow is induced causing relaxation and spreading of the chemical potential.

Figure 7 shows the analytical flow and numerical approximations in the plane of the crack. The dislocation causes flow over the crack surface and that increases toward the crack-tips.

To approximate the impulsive loading of the crack, a steep change in displacement was applied over a time length of $\Delta t$ and over a length interval $1 \pm \Delta x$. There is no difference in 


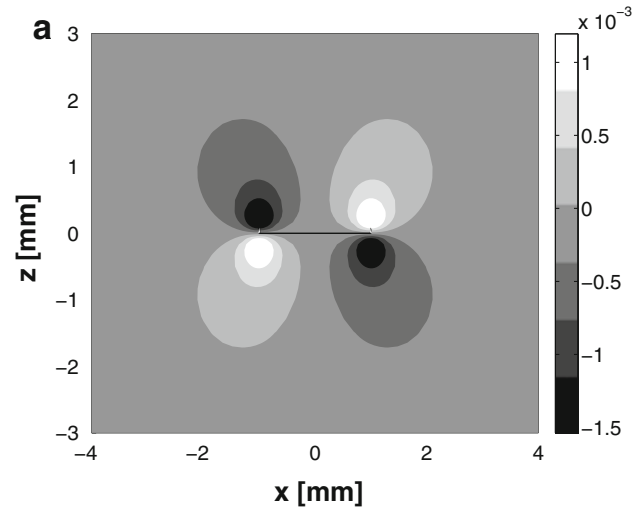

Fig. 6 Analytically calculated distribution of chemical potential (in $\mathrm{MPa}$ ) at $\mathbf{a} t=30.0 \mathrm{~s}$ and $\mathbf{b}$ at $t=301.1 \mathrm{~s}$. Crack is indicated by solid line. Fields of high and low chemical potential values are seen

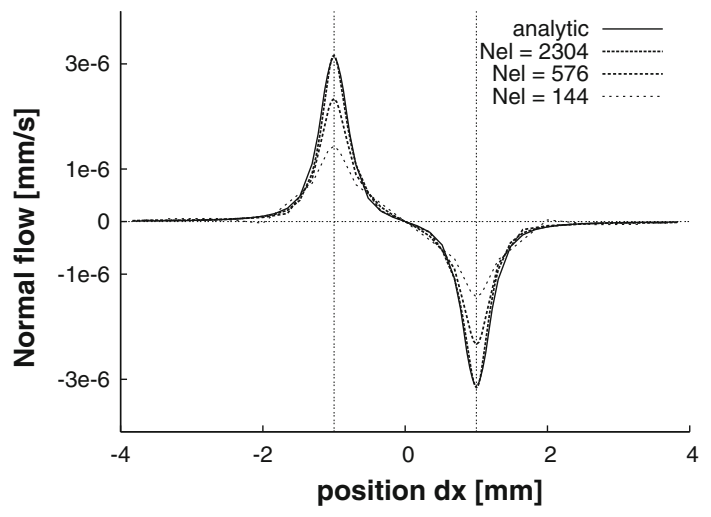

Fig. 7 The numerically and analytically calculated flow over the crack surface (in $\mathrm{mm} / \mathrm{s}$ ) at $t=30.0 \mathrm{~s}$. With refinement the numerically calculated flow converges to the analytical flow

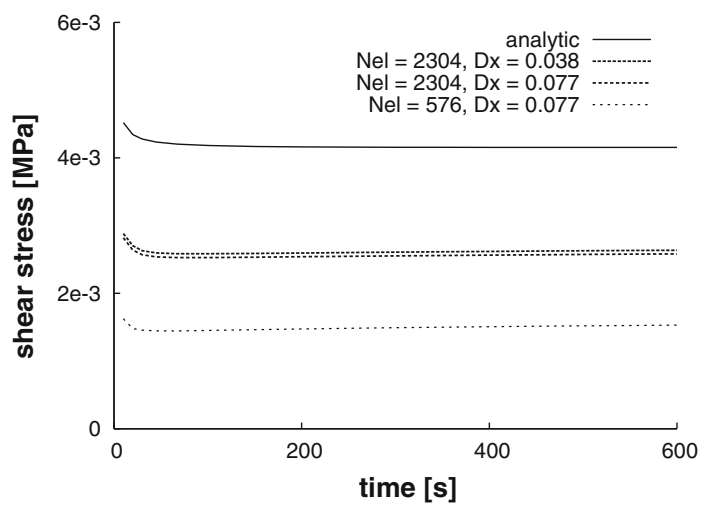

Fig. 8 Consolidation of shear stress at $x=1.0769 \mathrm{~mm}$. Refinement has a much larger effect than increasing interval length for spatial loading $\Delta x$ on the equilibrium stress

result between $\Delta t=0.1 \mathrm{~s}$ and $\Delta t=0.01 \mathrm{~s}$. The influence of the loading gradient is subsequently considered negligible for all calculations. Similarly in space, the Heaviside was approximated by prescribed nodal displacements along the crack, Fig. 3, up to an interval $\Delta x$ around the crack-tip. In

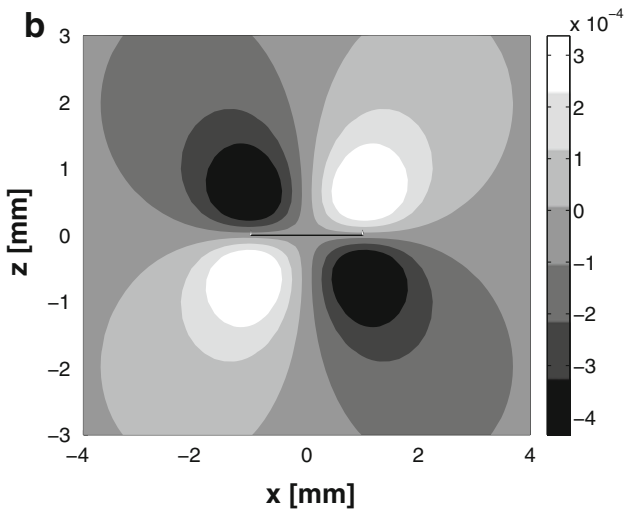

in regions of compression and extension respectively. The chemical potential relaxes and diffuses over time

Fig. 8, it is seen that refinement increased the overall accuracy and not so much the length of the unconstraint crack-tip region.

When the time distribution, as in Figs. 4 and 8, is considered more closely, it is clear that the numerical model has not reached equilibrium yet. Using that the stress at equilibrium satisfies Eq. (27), it is found that the analytical also has not reached equilibrium yet.

The osmotic prestress influences the initial shear stress as the result of incompressible behavior, but it influences the time to equilibrium and shear stress at equilibrium too. A decrease in osmotic pressure causes a decrease in shear stress, Fig. 9. Numerically this effect is smaller than analytically.

Numerical and analytical chemical potential distributions yield an overall good match. Analytically, at time $30.0 \mathrm{~s} \mathrm{max-}$ imum and minimum chemical potential of $\pm 1.50 \mathrm{e}-3 \mathrm{MPa}$ is found while numerically the chemical potential is a bit higher: $\pm 1.58 \mathrm{e}-3 \mathrm{MPa}$ for 2304 elements and $\pm 1.61 \mathrm{e}$ $3 \mathrm{MPa}$ for 576 elements. At time $301.1 \mathrm{~s}$ these values are $\pm 4.18 \mathrm{e}-4 \mathrm{MPa}$ for the analytical minima and maxima and $\pm 3.88 \mathrm{e}-4 \mathrm{MPa}$ for the numerical minima and maxima for 2304 elements and $\pm 4.04 \mathrm{e}-4 \mathrm{MPa}$ for 576 elements.

\section{Discussion}

In this research, an analytical solution for cracks in swelling porous media has been derived by considering a displacement driven dislocation. The dislocation is impulsively induced. The analytical solution has been derived by means of stress functions and Fourier and Laplace transformations. The solution is derived with physical units (i.e. not dimensionless) and converges to Booker's solution when there is no osmotic swelling. Minor differences between Booker's equations and our equations were found. For instance, it is unclear why Eqs. (19) and (20) and the general solutions are not completely consistent with Booker (1974), in 


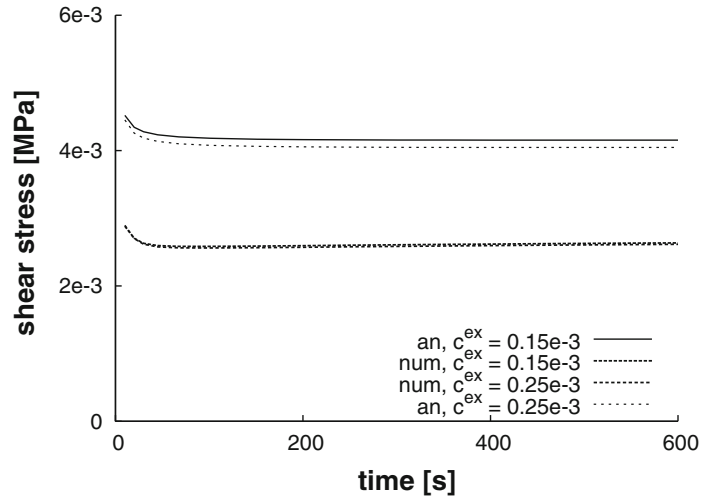

Fig. 9 Shear stress evolution at $x=1.0769 \mathrm{~mm}$ with $c_{e x}$ in $\mathrm{mmol} / \mathrm{mm}^{3}$. A decrease in prestress decreases the shear stress analytically and numerically, but increases the time to equilibrium. The analytical change in shear stress is larger than the numerical change, but smaller than the difference between analytical and numerical results

between Eqs. (12) and (13). Again for Eq. 23 there are minor unexplainable differences between our solutions and Booker (1974, Eq. (A1)). As none of the discrepancies propagate through the successive equations, these differences should probably be explained as typos in Booker's paper.

In the derivation of the analytical solution, some important assumptions were made. A small perturbation is considered, therefore $d \ll L$ must hold. The perturbation solution is additive to the initial state by linearization. This means that the initial state has a low influence. The jump over the $z=0$-plane of the dependent and independent variables are the boundary conditions over the crack. The displacement jump in shear direction is given, and other variables are continuous. This means that the shear stress response below and above the crack is symmetric about the $x=0$ plane. Another assumption is that far away from the crack the influence of the displacement at the crack is negligible.

In general, the decoupling method works well and can be used to consider other problems. The restriction of this method lies in the boundary conditions. Using these decoupled equations in Fourier domain, it is not possible to calculate the shear stress distribution in case of a semiinfinite crack. A Wiener-Hopf procedure can not be applied like models decoupled in drained and undrained variables (Atkinson and Craster 1991). Back transformation by numerical integration cannot be applied either.

The shear stress distribution in plane of the crack and the distribution in chemical potential have been derived in full dimensions including the osmotic prestress. From these solutions it is possible to derive a few properties. The shear stress undergoes a singularity of the order $\mathcal{O}\left(\frac{1}{\left(L^{2}-x^{2}\right)}\right)$ and relaxes exponentially (Eq. 25), but the high singularity at the cracktips does not vanish. The chemical potential distribution is not singular, see Eq. (29). In the crack the chemical potential is zero. In time the chemical potential decreases exponentially, but also the extremes of the pressure field move away from the crack-tips in time parallel to the $z$-axis (the factor $\mathrm{e}^{-z^{2} / 4 c K t}$ ). The tangential flow along the crack is zero. The normal flow in the $z=0$-plane, Eq. (30), has a singularity of the order $\mathcal{O}\left(\frac{1}{\left(L^{2}-x^{2}\right)}\right)$. The flow is positive for $x>0$ (upwards) and negative for $x<0$ (downwards), and zero for $x=0$.

The effect of parameters is apparent from Eq. (25). First, the stress is linearly dependent on the magnitude of the dislocation, but nonlinearly dependent on the length of the crack. The longer the crack the lower the shear stress produced by the dislocation, also in equilibrium. In general the term $\mu K F(x, t)$ is initially not small, but decreases in time to $\mu L / c$.

Secondly, higher Lamé constants result in increasing shear stress. The shear stress distribution is initially nonlinear in the shear modulus but in equilibrium it is linear. A higher Lamé constant $\lambda$ results in faster relaxation.

Finally, the response depends on external and internal ion concentrations. Increasing the external salt concentration decreases the prestress and decreases the shear stress response to the dislocation ( $C 1$ decreases and therefore $c$ decreases). The relaxation time is increased. Increasing the fixed charge concentration has the opposite reaction ( $c$ increases).

The analytical solution points out weaknesses of the numerical model. The numerical modeling has been validated by confined and unconfined pressure tests (Wilson et al. 2005b). From the expression for the chemical potential Eq. (29), the mesh size was determined such that the chemical potential is zero at the boundaries for the time frame of interest. The boundary conditions have little influence on the local shear stress distribution as long as the shear stress is constant around the area of interest. While the analytical solution assumes an unrestricted domain, numerically the domain is restricted. The restriction of the domain does have an influence on the solution, but comparison with a domain twice as large showed the influence is negligible. The differences of the numerical and analytical maxima and minima in the distribution of the chemical potential are the result of the use of a coarse mesh away from the area of interest. Furthermore, Fig. 7 shows that the finest mesh can capture the fluid flow very well.

Keeping the crack-tip node unrestricted has lead to a perfectly antisymmetric shear stress just as the analytical results, i.e. the shear stress left and right from the crack-tips are equal. Since the influence of time and spatial interval for reaching maximum values is low (Fig. 8), it is reasonable to use this unrestricted node. Furthermore, continuity in stresses holds, the stresses at the lower crack surface are equal to those at the upper crack surface.

The numerical model approximates the analytical solution very well, but in the very vicinity of the crack-tips the 
shear stress is underestimated by a factor 2, Fig. 5 . The time dependency of the numerical results matches the analytical solution very well for the finest mesh except for an offset at a point ahead of the crack-tip and close to the singularity (4). It is found that the time behavior is even better at the current crack surface. Considering the steepness of the spatial curve close to the singularity (Fig. 5), a slight offset in horizontal plane would cause a large mismatch in Fig. 4. Therefore, the offset in Fig. 4 should be interpreted as a minute horizontal shift of the numerical curves of Fig. 5 toward the singularity.

The importance of a good mesh and crack insertion is further emphasized when the effect of the osmotic prestress is considered, Fig. 9. The offset in the numerical calculations is larger than the change in shear stress perturbation as result of the change in osmotic prestress in absolute sense. The offset due to the horizontal shift of the numerical curve toward the singularity dominates other effects. In general, the influence of osmotic prestress for shearing mode is expected to be low, but is not negligible, Eq. (27). These effects could be overlooked if cracks are not treated correctly. A greater influence of the osmotic prestress is expected when considering the opening mode (Wognum et al. 2006). The analytical solution is a benchmark for the performance of numerical models with singularities and is a step toward arbitrary loading.

Acknowledgments The authors acknowledge financial support from the Technology Foundation STW, the technological branch of the Netherlands Organisation of Scientific Research NWO and the Ministry of Economic Affairs (DLR5790).

Open Access This article is distributed under the terms of the Creative Commons Attribution Noncommercial License which permits any noncommercial use, distribution, and reproduction in any medium, provided the original author(s) and source are credited.

\section{Appendix: Derivations for analytical solution}

\subsection{Derivation of stress functions}

The system is given by (32) and corresponds with (6).

$\mu \nabla^{2} u+\left(\lambda+\mu+R T \Gamma C_{1}\right) \frac{\partial}{\partial x} e-\frac{\partial}{\partial x} \mu^{\mathrm{f}}=0$,

$\mu \nabla^{2} w+\left(\lambda+\mu+R T \Gamma C_{1}\right) \frac{\partial}{\partial z} e-\frac{\partial}{\partial z} \mu^{\mathrm{f}}=0$,

$\frac{\partial e}{\partial t}-K \nabla^{2} \mu^{\mathrm{f}}=0$.

in which $e=\operatorname{tr}\left(\epsilon-\epsilon_{i}\right)$.

\subsubsection{Stress function $S$}

First, the stress function $S$ is derived, which is related to chemical potential. For this purpose, the first equation of (32) is differentiated with respect to $x$, the second with respect to $z$. The differentiated equations are then summed,

$\nabla^{2}\left(\left(2 \mu+\lambda+R T \Gamma C_{1}\right) e-\mu^{\mathrm{f}}-R T \Gamma C_{0}\right)=0$.

Following McNamee and Gibson (1960), the harmonic function $S(x, z, t)$ is introduced. If the chemical potential

$\mu^{\mathrm{f}}=\left(2 \mu+\lambda+R T \Gamma C_{1}\right) e-a \mu \frac{\partial S}{\partial z}-R T \Gamma C_{0}$.

then Eq. (33) is automatically satisfied if $S$ is harmonic. The value of the constant $a$ in (34) is chosen in the next steps. Equations (32) are reduced to

$\nabla^{2} u-\frac{\partial}{\partial x} e+a \frac{\partial^{2} S}{\partial x \partial z}=0$,

$\nabla^{2} w-\frac{\partial}{\partial z} e+a \frac{\partial^{2} S}{\partial z^{2}}=0$,

$\frac{\partial e}{\partial t}-K \nabla^{2} \mu^{\mathrm{f}}=0$.

The displacements are splitted in 3 components:

$u=u_{1}+u_{2}+u_{3}$,

$w=w_{1}+w_{2}+w_{3}$.

$u_{1}, w_{1}$ are associated with strain $e, u_{2}, w_{2}$ are associated with harmonic function $S$ and $u_{3}, w_{3}$ are harmonic functions. If these functions satisfy

$\nabla^{2} u_{1}+\frac{\partial e}{\partial x}=0$,

$\nabla^{2} u_{2}+a \frac{\partial^{2} S}{\partial x \partial z}=0$,

$\nabla^{2} u_{3}=0$

and

$\nabla^{2} w_{1}+\frac{\partial e}{\partial z}=0$,

$\nabla^{2} w_{2}+a \frac{\partial^{2} S}{\partial z^{2}}=0$

$\nabla^{2} w_{3}=0$

then the first two equations of (35) are satisfied. If we differentiate (37a) with respect to $x$ and (38a) with respect to $z$, one finds

$\nabla^{2} e=\nabla^{2}\left(\frac{\partial u_{1}}{\partial x}+\frac{\partial w_{1}}{\partial z}\right)$,

The factor $a$ is chosen $a=2$. This prevents fractions in the solution.

\subsubsection{Stress function E}

The solutions of Eqs. (37), (38) and (39) are given by (41). From these solutions the stress function $E=X+Y$ is derived, in which $X$ is an arbitrary differentiable function 
complying with $e=\nabla^{2} X$ and $Y$ is a harmonic function. The remaining equations are found through solving the PDEs. For instance, the solutions $u_{2}$ and $w_{2}$, related to harmonic function $S$, both follow the general solution (constant solution not taken into account)

$$
\begin{aligned}
u_{2}= & a_{1} S+a_{2} z S+a_{3} \frac{\partial S}{\partial x}+a_{4} \frac{\partial S}{\partial z} \\
& +a_{5} z \frac{\partial S}{\partial x}+a_{6} z \frac{\partial S}{\partial z}+\text { h.o.t. } \\
w_{2}= & b_{1} S+b_{2} z S+b_{3} \frac{\partial S}{\partial x}+b_{4} \frac{\partial S}{\partial z} \\
& +b_{5} z \frac{\partial S}{\partial x}+b_{6} z \frac{\partial S}{\partial z}+\text { h.o.t. }
\end{aligned}
$$

These terms are the result of the fact that $\nabla^{2} S=0$ holds. They are present in the general solutions for $u_{1}, u_{3}, w_{1}$ and $w_{3}$ as well. Furthermore, $a_{4}=-c_{2} / 2$ has to hold for $u_{2}$ and $b_{6}=-a / 2$ for $w_{2}$ to satisfy the second equations of (37) and (38). That is why $a=2$ was chosen. Equation (39) require $a_{1}=-b_{1}, a_{4}=-b_{3}, a_{3}=0, b_{4}=a / 2$. The solutions are

$$
\begin{aligned}
& e=\nabla^{2} X, \quad \nabla^{2} Y=0, \\
& u_{1}=\frac{\partial X}{\partial x}, u_{2}=-z \frac{\partial S}{\partial x}, \quad u_{3}=+\frac{\partial Y}{\partial x}, \\
& w_{1}=\frac{\partial X}{\partial z}, w_{2}=S-z \frac{\partial S}{\partial z}, w_{3}=+\frac{\partial Y}{\partial z} .
\end{aligned}
$$

7.2 State variables in terms of stress functions

$$
u=\frac{\partial E}{\partial x}-z \frac{\partial S}{\partial x},
$$

$w=\frac{\partial E}{\partial z}-z \frac{\partial S}{\partial z}+S$,

$\mu^{\mathrm{f}}=c \nabla^{2} E-2 \mu \frac{\partial S}{\partial z}$,

The stresses are given by

$$
\begin{aligned}
& \sigma_{x x}=-2 \mu\left\{\frac{\partial^{2} E}{\partial z^{2}}+z \frac{\partial^{2} S}{\partial x^{2}}-\frac{\partial S}{\partial z}\right\} \\
& \sigma_{x z}=2 \mu\left\{\frac{\partial^{2} E}{\partial x \partial z}-z \frac{\partial^{2} S}{\partial x \partial z}\right\} \\
& \sigma_{z z}=-2 \mu\left\{\frac{\partial^{2} E}{\partial x^{2}}+z \frac{\partial^{2} S}{\partial z^{2}}-\frac{\partial S}{\partial z}\right\}
\end{aligned}
$$

These are used to derive the boundary conditions.

\subsection{Jump condition mode II}

Shear faulting is considered. For shear faulting the displacement jump between $z=0^{-}$and $z=0^{+}$is given by

$[u]=f(x) \mathcal{H}(t)$. with $f(x)$ the slip function, and $\mathcal{H}(t)$ the Heaviside function. There is no jump in normal displacement and therefore $[\hat{w}]=0$ holds at $z=0$. The slip function has Fourier transform $\tilde{f}(k)$. Then, taking Fourier and Laplace transformation gives at $z=0$ :

$$
\begin{aligned}
& {[\hat{u}]=\tilde{f}(k) / p=i k \hat{E} \rightarrow[\hat{E}]=\frac{\tilde{f}(k)}{i k p} .} \\
& {[\hat{w}]=0 \rightarrow\left[\frac{\partial \hat{E}}{\partial z}\right]=-[\hat{S}] .}
\end{aligned}
$$

Other jump conditions at $z=0$ is continuity of pore pressure and its normal derivative:

$$
\begin{aligned}
& {\left[\hat{\mu^{\mathrm{f}}}\right]=0 . \rightarrow c\left[\frac{\partial^{2} \hat{E}}{\partial z^{2}}\right]-c k^{2}[\hat{E}]-2 \mu\left[\frac{\partial \hat{S}}{\partial z}\right]=0 .} \\
& {\left[\frac{\partial \hat{\mu^{\mathrm{f}}}}{\partial z}\right]=0 . \rightarrow c\left[\frac{\partial^{3} \hat{E}}{\partial z^{3}}\right]-c k^{2}\left[\frac{\partial \hat{E}}{\partial z}\right]-2 \mu\left[\frac{\partial^{2} \hat{S}}{\partial z^{2}}\right]=0 .}
\end{aligned}
$$

Furthermore, continuity of stresses $\hat{\sigma}_{x z}$ and $\hat{\sigma}_{z z}$ is assumed.

$\left[\hat{\sigma}_{x z}\right]=\left[\hat{\sigma}_{z z}\right]=0$.

The following holds:

$$
\begin{aligned}
& 2 \mu i k\left\{\left[\frac{\partial \hat{E}}{\partial z}\right]\right\}=0 . \\
& -2 \mu\left\{-k^{2}[\hat{E}]-\left[\frac{\partial \hat{S}}{\partial z}\right]\right\}=0 .
\end{aligned}
$$

Equations (45)-(49) form six equations with seven unknowns. They reduce to jump conditions for the stress functions and their derivatives and for the transformed equation (65).

The first jump condition is derived from (45).

$$
[\hat{E}]=\frac{-i}{k s} \tilde{f}(k) \text {, }
$$

while (49) gives

$$
\left[\frac{\partial \hat{E}}{\partial z}\right]=0 .
$$

Substituting (50) in (49) results in jump condition

$\left[\frac{\partial \hat{S}}{\partial z}\right]=\frac{i k}{s} \tilde{f}(k)$

Substituting (51) in (46) results in the next jump condition

$[\hat{S}]=0$.

Inserting the jump conditions (50) and (52) in Eq. (47) gives the jump condition for second-order derivative of 


\section{function $E$}

$\left[\frac{\partial^{2} \hat{E}}{\partial z^{2}}\right]=-\frac{i k}{s} \tilde{f}(k)(1-2 \mu / c)$.

The last jump condition is derived by inserting the jump condition (51) in Eq. (47).

$$
\left[\frac{\partial^{2} \hat{S}}{\partial z^{2}}\right]=\frac{c}{2 \mu}\left[\frac{\partial^{3} \hat{E}}{\partial z^{3}}\right] \text {. }
$$

\subsection{Fourier and Laplace transformations}

Integral forms are used to handle discontinuities in space and time. Another advantage is that integration and differentiation become multiplication and division. We define the next Fourier and Laplace transformations of the decoupling functions $E$ and $S$.

$\tilde{E}(k, z, t)=\int_{-\infty}^{\infty} E(x, z, t) \mathrm{e}^{-i k x} \mathrm{~d} x$

$\hat{E}(k, z, s)=\int_{0}^{\infty} \tilde{E}(k, z, t) \mathrm{e}^{-s t} \mathrm{~d} t$

$\tilde{S}(k, z, t)=\int_{-\infty}^{\infty} S(x, z, t) \mathrm{e}^{-i k x} \mathrm{~d} x$

$\hat{S}(k, z, s)=\int_{0}^{\infty} \tilde{S}(k, z, t) \mathrm{e}^{-s t} \mathrm{~d} t$

\subsubsection{Fourier transformations}

$\int_{-\infty}^{\infty}\left\{\frac{\partial E}{\partial x}\right\} \mathrm{e}^{-i k x} \mathrm{~d} x=\int_{-\infty}^{\infty} \mathrm{e}^{-i k x} \mathrm{~d} E$

$$
=i k \tilde{E} \text {, }
$$

$\int_{-\infty}^{\infty}\left\{\frac{\partial^{2} E}{\partial x^{2}}\right\} \mathrm{e}^{-i k x} \mathrm{~d} x=\int_{-\infty}^{\infty} \mathrm{e}^{-i k x} \mathrm{~d}\left(\frac{\partial E}{\partial x}\right)$

$$
=-k^{2} \tilde{E},
$$

$\int_{-\infty}^{\infty}\left\{\nabla^{2} E\right\} \mathrm{e}^{-i k x} \mathrm{~d} x=\left(\frac{\partial^{2}}{\partial z^{2}}-k^{2}\right) \tilde{E}$.

Concluding, it holds that

$\tilde{e}=\left(\frac{\partial^{2}}{\partial z^{2}}-k^{2}\right) \tilde{E}$.

Then, for the displacement functions and pore pressure one finds

$$
\begin{aligned}
\tilde{u} & =\int_{-\infty}^{\infty} u(x, z, t) \mathrm{e}^{-i k x} \mathrm{~d} x \\
& =\int_{-\infty}^{\infty}\left(\frac{\partial E}{\partial x}-z \frac{\partial S}{\partial x}\right) \mathrm{e}^{i k x} \mathrm{~d} x \\
& =i k(\tilde{E}-z \tilde{S}) . \\
\tilde{w} & =\int_{-\infty}^{\infty} w(x, z, t) \mathrm{e}^{-i k x} \mathrm{~d} x \\
& =\int_{-\infty}^{\infty}\left(\frac{\partial E}{\partial z}-z \frac{\partial S}{\partial z}+S\right) \mathrm{e}^{i k x} \mathrm{~d} x \\
& =\frac{\partial \tilde{E}}{\partial z}-z \frac{\partial \tilde{S}}{\partial z}+\tilde{S} . \\
\tilde{\mu^{\mathrm{f}}} & =c\left(\frac{\partial^{2}}{\partial z^{2}}-k^{2}\right) \tilde{E}-2 \mu \frac{\partial \tilde{S}}{\partial z}-R T \Gamma C_{0} 2 \pi \delta(k) .
\end{aligned}
$$

The Fourier transformed stress components become

$$
\begin{aligned}
& \tilde{\sigma}_{x x}=-2 \mu\left\{\frac{\partial^{2} \tilde{E}}{\partial z^{2}}-z k^{2} \tilde{S}-\frac{\partial \tilde{S}}{\partial z}\right\} . \\
& \tilde{\sigma}_{x z}=2 \mu i k\left\{\frac{\partial \tilde{E}}{\partial z}-z \frac{\partial \tilde{S}}{\partial z}\right\} . \\
& \tilde{\sigma}_{z z}=-2 \mu\left\{-k^{2} \tilde{E}+z \frac{\partial^{2} \tilde{S}}{\partial z^{2}}-\frac{\partial \tilde{S}}{\partial z}\right\}
\end{aligned}
$$

The Fourier transformed governing equations become

$$
\begin{aligned}
& \left(\frac{\partial^{2}}{\partial z^{2}}-k^{2}\right) \tilde{S}=0 \\
& \left(\frac{\partial^{2}}{\partial z^{2}}-k^{2}\right) \frac{\partial \tilde{E}}{\partial t}-c K\left(\frac{\partial^{2}}{\partial z^{2}}-k^{2}\right)^{2} \tilde{E}=0 .
\end{aligned}
$$

\subsection{Laplace transformations}

To take out the time dependency also Laplace transformation is performed.

$$
\int_{0}^{\infty} \frac{\partial \tilde{E}}{\partial t} \mathrm{e}^{-s t} \mathrm{~d} t=\int_{0}^{\infty} \mathrm{e}^{-s t} \mathrm{~d} \tilde{E}=+s \hat{E} .
$$

The Laplace transformed equations for displacements (59), stress components (60) and governing equations (61) are (63) to (65). 
$\hat{u}=i k(\hat{E}-z \hat{S})$

$\hat{w}=\frac{\partial}{\partial z} \hat{E}-\left(z \frac{\partial}{\partial z}-1\right) \hat{S}$,

$\hat{\mu^{\mathrm{f}}}=c\left(\frac{\partial^{2}}{\partial z^{2}}-k^{2}\right) \hat{E}-2 \mu \frac{\partial}{\partial z} \hat{S}-R T \Gamma C_{0} \frac{2 \pi \delta(k)}{s}$.

$\hat{\sigma}_{x x}=-2 \mu\left\{\frac{\partial^{2}}{\partial z^{2}} \hat{E}-\left(z k^{2}+\frac{\partial}{\partial z}\right) \hat{S}\right\}$.

$\hat{\sigma}_{x z}=2 \mu i k\left\{\frac{\partial}{\partial z} \hat{E}-z \frac{\partial}{\partial z} \hat{S}\right\}$.

$\hat{\sigma}_{z z}=-2 \mu\left\{-k^{2} \hat{E}+\left(z \frac{\partial^{2}}{\partial z^{2}}-\frac{\partial}{\partial z}\right) \hat{S}\right\}$

$\left(\frac{\partial^{2}}{\partial z^{2}}-k^{2}\right) \hat{S}=0$

$\left(\frac{\partial^{2}}{\partial z^{2}}-k^{2}\right)\left(c K\left(\frac{\partial^{2}}{\partial z^{2}}-k^{2}\right)-s\right) \hat{E}=0$.

\subsection{Laplace solution}

Because the impact of the crack fades away far from the crack, the solution has to approach zero for $z \rightarrow \pm \infty$. The domain is divided into an upper half plane and a lower half plane, because there is a sign dependence.

\subsubsection{Solution of $\hat{S}$}

First, the solution of the harmonic equation (65a) is derived. The problem is given by

$$
\begin{aligned}
& \left(\frac{\partial^{2}}{\partial z^{2}}-k^{2}\right) \hat{S}=0 \\
& {[\hat{S}]=0 \quad\left[\frac{\partial \hat{S}}{\partial z}\right]=\frac{i k}{s} \tilde{f}(k) .}
\end{aligned}
$$

The general solution of this Laplace equation is given by

$$
\hat{S}(k, z, s)=a_{1} \mathrm{e}^{-k z}+a_{2} \mathrm{e}^{+k z} .
$$

For $z>0$ the solution is given by $\hat{S}_{+}=a_{+} \mathrm{e}^{-|k| z}$ and for $z<0$ the solution is given by $\hat{S}_{-}=a_{-} \mathrm{e}^{+|k| z}$. As jump conditions, the jump over the displacement is given for the stress function $S$ and its derivative.

$$
\begin{aligned}
& \lim _{z \downarrow 0} \hat{S}_{+}-\lim _{z \uparrow 0} \hat{S}_{-}=a_{+}-a_{-}=0 \\
& \lim _{z \downarrow 0} \frac{\partial \hat{S}_{+}}{\partial z}-\lim _{z \uparrow 0} \frac{\partial \hat{S}_{-}}{\partial z}=-|k|\left(a_{+}+a_{-}\right)=\frac{i k}{s} \tilde{f}(k) .
\end{aligned}
$$

With $\operatorname{sign}(k)=k /|k|$ the next holds

$a_{+}=a_{-}=\frac{-i \operatorname{sign}(k)}{2 s} \tilde{f}(k)$.

In conclusion, the solution of (66) is given by

$$
\hat{S}(k, z, s)=\frac{-i \operatorname{sign}(k)}{2 s} \tilde{f}(k) \mathrm{e}^{-|k||z|} .
$$

Note that $\left[\frac{\partial^{2} \hat{S}}{\partial z^{2}}\right]=k^{2} a_{+}-k^{2} a_{-}=0$.

\subsubsection{Solution of $\hat{E}$}

The mass equation is solved similarly to the harmonic stress function. The problem is

$$
\begin{aligned}
& \left(\frac{\partial^{2}}{\partial z^{2}}-k^{2}\right)\left(c K\left(\frac{\partial^{2}}{\partial z^{2}}-k^{2}\right)-s\right) \hat{E}=0 . \\
& {[\hat{E}]=\frac{-i}{k s} \tilde{f}(k) \quad\left[\frac{\partial \hat{E}}{\partial z}\right]=0,} \\
& {\left[\frac{\partial^{2} \hat{E}}{\partial z^{2}}\right]=-\frac{i k}{c s} \tilde{f}(k)(c-2 \mu)\left[\frac{\partial^{3} \hat{E}}{\partial z^{3}}\right]=0 .}
\end{aligned}
$$

The general solution is given by (72) with $\gamma=\sqrt{k^{2}+\frac{s}{c K}}$ and $\Re(\gamma)>0$. The solution is again divided into one for the upper half plane and one for the lower half plane.

$$
\hat{E}(k, z, s)=a_{+} \mathrm{e}^{-k z}+a_{-} \mathrm{e}^{+k z}+b_{+} \mathrm{e}^{-\gamma z}+b_{-} \mathrm{e}^{\gamma z} .
$$

Again the jump conditions are used to determine these coefficients. $a_{+}=-a_{-}$and $b_{+}=-b_{-}$hold. Then, the solution of (72) is given by (73)

$$
\hat{E}(k, z, s)=a_{+} \operatorname{sign}(z) \mathrm{e}^{-|k||z|}+b_{+} \operatorname{sign}(z) \mathrm{e}^{-\gamma|z|} .
$$

with

$a_{+}=-\left(\frac{i k \tilde{f}(k) \mu K}{s^{2}}+\frac{i \tilde{f}(k)}{2 k s}\right)$,

$b_{+}=\frac{i k \mu \tilde{f}(k) K}{s^{2}}$

Then, in total, the solution is given by

$$
\begin{aligned}
\hat{E}(k, z, s)= & -\operatorname{sign}(z)\left\{\frac{i k \mu \tilde{f}(k) K}{s^{2}}+\frac{i \tilde{f}(k)}{2 k s}\right\} \mathrm{e}^{-|k||z|} \\
& +\operatorname{sign}(z)\left\{\frac{i k \mu \tilde{f}(k) K}{s^{2}}\right\} \mathrm{e}^{-\frac{|z|}{\sqrt{c K}} \sqrt{k^{2} c K+s}}
\end{aligned}
$$

We introduce the Fourier transformed function $\tilde{g}(k)$ such that

$\tilde{g}(k)=i k \mu \tilde{f}(k) K$

Solution rewritten becomes then

$$
\begin{aligned}
\hat{E}(k, z, s)= & \operatorname{sign}(z)\left\{\left[-\frac{\tilde{g}(k)}{s^{2}}-\frac{i \tilde{f}(k)}{2 k s}\right] \mathrm{e}^{-|k||z|}\right. \\
& \left.+\frac{\tilde{g}(k)}{s^{2}} \mathrm{e}^{-\frac{|z|}{\sqrt{c K}} \sqrt{k^{2} c K+s}}\right\} .
\end{aligned}
$$




\subsection{Inverse Laplace}

\subsubsection{Inverse stress function $\hat{S}$}

When the solution of transformed equations are considered, several terms dependent on time are present. For the stress function $\hat{S}$, the inverse Laplace is rather simple to derive. $\hat{S}$ contains a factor $1 / s$. The inverse Laplace is given by

$\mathcal{L}^{-1}\left(\frac{1}{s}\right)=\mathcal{H}(t)$

The inverse Laplace of (70) for the stress function $\hat{S}$ is

$\tilde{S}(k, z, t)=-\frac{i \operatorname{sign}(k)}{2} \tilde{f}(k) \mathrm{e}^{-|k||z|} \mathcal{H}(t)$.

\subsubsection{Inverse stress function $\hat{E}$}

The inverse Laplace of stress function $\hat{E}$ is more difficult. It contains factors $1 / s, 1 / s^{2}$ but also factor $\frac{\mathrm{e}^{-\frac{|z| \sqrt{k^{2} c K+s}}{\sqrt{c K}}}}{s^{2}}$. This latter term is not easy to invert. The next holds.

$\mathcal{L}^{-1}\left(\frac{1}{s^{2}}\right)=t \mathcal{H}(t)$

For deriving the inverse of the complex term, the shift theorem is needed and the convolution theorem. The next steps are followed

1. The exponent is inversed

$$
\mathcal{L}^{-1}\left(\mathrm{e}^{-\frac{|z|}{\sqrt{c K}} \sqrt{s}}\right)=\frac{|z|}{\sqrt{c K} \sqrt{4 \pi t^{3}}} \mathrm{e}^{-\frac{z^{2}}{4 c k t}} .
$$

2. Shift theorem $\mathcal{L}^{-1}(F(s-a))=\mathrm{e}^{a t} f(t)$ is used:

$$
\mathcal{L}^{-1}\left(\mathrm{e}^{-\frac{|z|}{\sqrt{c K}} \sqrt{k^{2} c K+s}}\right)=\frac{|z|}{\sqrt{c K} \sqrt{4 \pi t^{3}}} \mathrm{e}^{-\frac{z^{2}}{4 c k t}-k^{2} c K t} .
$$

3. Convolution theorem $\mathcal{L}^{-1}(F G)=f * g=\int g(t-\tau)$ $f(\tau) d \tau$ is used:

$$
\begin{aligned}
& \mathcal{L}^{-1}\left(\frac{\mathrm{e}^{-\frac{|z|}{\sqrt{c K}} \sqrt{k^{2} c K+s}}}{s^{2}}\right) \\
& =\int_{0}^{t} \frac{|z|(t-\tau)}{\sqrt{c K} \sqrt{4 \pi \tau^{3}}} \mathrm{e}^{-\frac{z^{2}}{4 c k \tau}-k^{2} c K \tau} \mathrm{d} \tau .
\end{aligned}
$$

The integration in Eq. (83) is solved by substitution of $x^{2}=\tau(2 x d x=\mathrm{d} \tau)$ and substitution of $b=\frac{z^{2}}{4 c K}, a=$ $k^{2} c K$

$$
\begin{aligned}
\mathcal{L}^{-1}(\ldots) & =\int_{0}^{t} \frac{|z|(t-\tau)}{\sqrt{c K} \sqrt{4 \pi \tau^{3}}} \mathrm{e}^{-\frac{z^{2}}{4 c k \tau}-k^{2} c K \tau} \mathrm{d} \tau \\
& =\frac{|z|}{\sqrt{c K \pi}} \int_{0}^{\sqrt{t}}\left(\frac{t}{x^{2}}-1\right) \mathrm{e}^{-\frac{z^{2}}{4 c k x^{2}}-k^{2} c K x^{2}} \mathrm{~d} x \\
& =-\frac{|z|}{\sqrt{c K \pi}} \int_{0}^{\sqrt{t}}\left(1+t \frac{\partial}{\partial b}\right) \mathrm{e}^{-\frac{b}{x^{2}}-a x^{2}} \mathrm{~d} x, \\
& =-\frac{|z|}{\sqrt{c K \pi}}\left(1+t \frac{\partial}{\partial b}\right) \int_{0}^{\sqrt{t}} \mathrm{e}^{-\frac{b}{x^{2}}-a x^{2}} \mathrm{~d} x .
\end{aligned}
$$

This last integral can be solved by using the formula 7.4.33 of Abramowitz and Stegun (1970). The solution is a combination of error functions. Note that $b$ is positive and therefore the next holds,

$$
\begin{aligned}
\lim _{x \downarrow 0} \operatorname{erf}( & \sqrt{a} x-\sqrt{b} / x)=\lim _{x \downarrow 0} \operatorname{erf}(-\sqrt{b} / x)=-1, \\
\lim _{x \downarrow 0} \operatorname{erf}( & \sqrt{a} x+\sqrt{b} / x)=\lim _{x \downarrow 0} \operatorname{erf}(\sqrt{b} / x)=+1 . \\
\mathcal{L}^{-1}(\ldots)= & -\frac{|z|}{\sqrt{c K \pi}} \frac{\sqrt{\pi}}{4 \sqrt{a}}\left(1+t \frac{\partial}{\partial b}\right) \\
& \times\left[\mathrm{e}^{2 \sqrt{a b}} \operatorname{erf}(\sqrt{a} x+\sqrt{b} / x)\right. \\
& \left.+\mathrm{e}^{-2 \sqrt{a b}} \operatorname{erf}(\sqrt{a} x-\sqrt{b} / x)\right]_{0}^{\sqrt{t}} \\
= & -\frac{|z|}{\sqrt{c K}} \frac{1}{4 \sqrt{a}}\left(1+t \frac{\partial}{\partial b}\right) \\
& \times\left(\mathrm{e}^{2 \sqrt{a b}}(-1+\operatorname{erf}(\sqrt{a t}\right. \\
& \left.+\sqrt{b / t}))+\mathrm{e}^{-2 \sqrt{a b}}(1+\operatorname{erf}(\sqrt{a t}-\sqrt{b / t}))\right) \\
= & +\frac{|z|}{4 \sqrt{c K a}}\left(1+t \frac{\partial}{\partial b}\right) \\
& \times\left(\mathrm{e}^{2 \sqrt{a b}} \operatorname{erfc}(\sqrt{a t}+\sqrt{b / t})\right. \\
& \left.-\mathrm{e}^{-2 \sqrt{a b}}(2-\operatorname{erfc}(\sqrt{a t}-\sqrt{b / t}))\right)
\end{aligned}
$$

Considering that $\frac{\partial \operatorname{erfc}(x)}{\partial x}=-2 \mathrm{e}^{x^{2}} / \sqrt{\pi}$, we find

$$
\begin{aligned}
& \frac{\partial}{\partial b} \mathrm{e}^{2 \sqrt{a b}}=\frac{\sqrt{a}}{\sqrt{b}} \mathrm{e}^{2 \sqrt{a b}}, \\
& \frac{\partial}{\partial b} \operatorname{erfc}(\sqrt{a t}+\sqrt{b / t})=-\frac{1}{\sqrt{\pi b t}} \mathrm{e}^{-(\sqrt{a t}+\sqrt{b / t})^{2}} \\
& \frac{\partial}{\partial b} \mathrm{e}^{-2 \sqrt{a b}}=-\frac{\sqrt{a}}{\sqrt{b}} \mathrm{e}^{-2 \sqrt{a b}}, \\
& \frac{\partial}{\partial b} \operatorname{erfc}(\sqrt{a t}-\sqrt{b / t})=\frac{1}{\sqrt{\pi b t}} \mathrm{e}^{-(\sqrt{a t}-\sqrt{b / t})^{2}}
\end{aligned}
$$


Then the derivatives of the error functions become

$$
\begin{aligned}
& \frac{\partial}{\partial b}\left(\mathrm{e}^{2 \sqrt{a b}} \operatorname{erfc}(\sqrt{a t}+\sqrt{b / t})\right) \\
& =\sqrt{a / b} \mathrm{e}^{2 \sqrt{a b}} \operatorname{erfc}(\sqrt{a t}+\sqrt{b / t})-\frac{\mathrm{e}^{2 \sqrt{a b}}}{\sqrt{\pi b t}} \mathrm{e}^{-(\sqrt{a t}+\sqrt{b / t})^{2}} . \\
& \frac{\partial}{\partial b}\left(\mathrm{e}^{2 \sqrt{a b}} \operatorname{erfc}(\sqrt{a t}-\sqrt{b / t})\right) \\
& =-\sqrt{a / b} \mathrm{e}^{-2 \sqrt{a b}} \operatorname{erfc}(\sqrt{a t}-\sqrt{b / t})+\frac{\mathrm{e}^{-2 \sqrt{a b}}}{\sqrt{\pi b t}} \mathrm{e}^{-(\sqrt{a t}-\sqrt{b / t})^{2} .}
\end{aligned}
$$

And for the exponents holds

$$
\begin{aligned}
& \mathrm{e}^{-2 \sqrt{a b}} \mathrm{e}^{-(\sqrt{a t}-\sqrt{b / t})^{2}} \\
& \quad=\mathrm{e}^{2 \sqrt{a b}} \mathrm{e}^{-(\sqrt{a t}+\sqrt{b / t})^{2}}=\mathrm{e}^{-(a t+b / t)} .
\end{aligned}
$$

Then differentiating with respect to $b$ in Eq. (86), we find for the inverse Laplace of the exponent through convolution theory (Eq. (83)) and with $b=\frac{z^{2}}{4 c K}$ and $a=k^{2} c K$.

$$
\begin{aligned}
& \mathcal{L}^{-1}(\ldots)=\left(\frac{-|z|}{2|k| c K}+t\right) \mathrm{e}^{-|k||z|} \\
& +\left(\frac{|z|}{4|k| c K}+t / 2\right) \mathrm{e}^{+|k||z|} \operatorname{erf} c(|k| \sqrt{c K t}+|z| / \sqrt{4 c K t}) \\
& +\left(\frac{|z|}{4|k| c K}-t / 2\right) \mathrm{e}^{-|k||z|} \operatorname{erf} c(|k| \sqrt{c K t}-|z| / \sqrt{4 c K t}) .
\end{aligned}
$$

The Fourier transformed stress function $\tilde{E}$ becomes

$$
\begin{aligned}
\mathcal{L}^{-1}(\hat{E})= & \tilde{E}(k, z, t) \\
= & \operatorname{sign}(z)\left\{\left[-\tilde{g}(k) t-\frac{i \tilde{f}(k)}{2 k}\right] \mathrm{e}^{-|k||z|} \mathcal{H}(t)\right. \\
& \left.+\tilde{g}(k) \mathcal{L}^{-1}\left(\frac{\mathrm{e}^{-\frac{1}{c k} \sqrt{k^{2} c K+s}|z|}}{s^{2}}\right)\right\}
\end{aligned}
$$

with $\tilde{g}(k)=i k \mu \tilde{f}(k) K$. This expression for the stress function is reduced to

$$
\begin{aligned}
& \tilde{E}(k, z, t)=-\operatorname{sign}(z)\left\{\left[\frac{i \tilde{f}(k)}{2 k}+\frac{|z|}{2|k| c K} \tilde{g}(k)\right] \mathrm{e}^{-|k||z|} \mathcal{H}(t)\right. \\
& -\tilde{g}(k)\left(\frac{t}{2}+\frac{|z|}{4|k| c K}\right) \mathrm{e}^{|k||z|} \operatorname{erf} c(|k| \sqrt{c K t}+|z| / \sqrt{4 c K t}) \\
& \left.+\tilde{g}(k)\left(\frac{t}{2}-\frac{|z|}{4|k| c K}\right) \mathrm{e}^{-|k||z|} \operatorname{erf} c(|k| \sqrt{c K t}-|z| / \sqrt{4 c K t})\right\} .
\end{aligned}
$$

or rewritten

$$
\begin{aligned}
\tilde{E}(k, z, t)= & -\frac{i \mu \tilde{f}(k) \operatorname{sign}(z)}{2 c k}\left\{\left[\frac{c}{\mu}+|z||k|\right] \mathrm{e}^{-|k||z|} \mathcal{H}(t)\right. \\
& -c K\left(k^{2} t+\frac{|z||k|}{2 c K}\right) \mathrm{e}^{|k||z|} \operatorname{erf} c(|k| \sqrt{c K t} \\
& +|z| / \sqrt{4 c K t}) \\
& +c K\left(k^{2} t-\frac{|z||k|}{2 c K}\right) \mathrm{e}^{-|k||z|} \operatorname{erf} c(|k| \sqrt{c K t} \\
& -|z| / \sqrt{4 c K t})\} .
\end{aligned}
$$

\subsubsection{Derivatives of inverse stress functions}

A short notation is introduced:

$$
\begin{aligned}
& \operatorname{erf} c(|k| \sqrt{c K t}-|z| / \sqrt{4 c K t})=\operatorname{erfc}(-), \\
& \operatorname{erf} c(|k| \sqrt{c K t}+|z| / \sqrt{4 c K t})=\operatorname{erfc}(+) .
\end{aligned}
$$

Partial differentiation with respect to $z$ results in:

$$
\begin{aligned}
& \frac{\partial}{\partial z}\left(\mathrm{e}^{(-|k||z|)}\right)=-\operatorname{sign}(z)|k| \mathrm{e}^{(-|k||z|)} \\
& \frac{\partial}{\partial z}\left(\mathrm{e}^{(|k||z|)}\right)=\operatorname{sign}(z)|k| \mathrm{e}^{(|k||z|)}
\end{aligned}
$$

and

$\mathrm{e}^{( \pm|k||z|)} \frac{\partial}{\partial z}(\operatorname{erfc}( \pm))=-\frac{ \pm \operatorname{sign}(z)}{\sqrt{\pi c K t}} \mathrm{e}^{-\left(k^{2} c K t+z^{2} /(4 c K t)\right)}$.

The first-order derivatives with respect to $z$ of the stress functions are given below.

$$
\begin{aligned}
& \frac{\partial \tilde{E}}{\partial z}(k, z, t)=\left\{\left[\frac{i|k| \tilde{f}(k)}{2 k}+\frac{|z||k|-1}{2|k| c K} \tilde{g}(k)\right] \mathrm{e}^{-|k||z|} \mathcal{H}(t)\right. \\
& +\tilde{g}(k)\left(\frac{t|k|}{2}+\frac{|z||k|+1}{4|k| c K}\right) \mathrm{e}^{|k||z|} \operatorname{erfc}(+) \\
& +\tilde{g}(k)\left(\frac{t|k|}{2}-\frac{|z||k|-1}{4|k| c K}\right) \mathrm{e}^{-|k||z|} \operatorname{erfc}(-) \\
& \left.-\frac{\tilde{g}(k) t}{\sqrt{\pi c K t}} \mathrm{e}^{-\left(k^{2} c K t+z^{2} /(4 c K t)\right)}\right\} \text {. } \\
& =\frac{i \mu \tilde{f}(k)}{2 c k}\left\{|k|\left[\frac{c}{\mu}+|z||k|-1\right] \mathrm{e}^{-|k||z|} \mathcal{H}(t)\right. \\
& +|k| c K\left(k^{2} t+\frac{|z||k|+1}{2 c K}\right) \mathrm{e}^{|k||z|} \operatorname{erf} c(+) \\
& +|k| c K\left(k^{2} t-\frac{|z||k|-1}{2 c K}\right) \mathrm{e}^{-|k||z|} \operatorname{erfc}(-) \\
& \left.-2 \frac{k^{2} \sqrt{c K t}}{\sqrt{\pi}} \mathrm{e}^{-\left(k^{2} c K t+z^{2} /(4 c K t)\right)}\right\} \text {. } \\
& \frac{\partial \tilde{S}}{\partial z}(k, z, t)=\frac{i k \operatorname{sign}(z)}{2} \tilde{f}(k) \mathrm{e}^{-|k||z|} \mathcal{H}(t) .
\end{aligned}
$$


The second-order derivatives with respect to $z$ are

$$
\begin{aligned}
& \frac{\partial^{2} \tilde{E}}{\partial z^{2}}(k, z, t)=-\operatorname{sign}(z)\left\{\left[\frac{i k \tilde{f}(k)}{2}\right.\right. \\
& \left.+\frac{|z| k^{2}-2 \operatorname{sign}(z)|k|}{2|k| c K} \tilde{g}(k)\right] \mathrm{e}^{-|k||z|} \mathcal{H}(t) \\
& \quad-\tilde{g}(k)\left(\frac{t k^{2}}{2}+\frac{|z||k|+2 \operatorname{sign}(z)}{4 c K}\right) \mathrm{e}^{|k||z|} \operatorname{erf} c(+) \\
& \left.\quad+\tilde{g}(k)\left(\frac{t k^{2}}{2}-\frac{|z||k|-2 \operatorname{sign}(z)}{4 c K}\right) \mathrm{e}^{-|k||z|} \operatorname{erf} c(-)\right\} \\
& =-\frac{i \mu \tilde{f}(k) \operatorname{sign}(z)}{2 c k}\left\{k^{2}\left[\frac{c}{\mu}+|z||k|-2\right] \mathrm{e}^{-|k||z|} \mathcal{H}(t)\right. \\
& \quad-k^{2} c K\left(k^{2} t+\frac{|z||k|+2}{2 c K}\right) \mathrm{e}^{|k||z|} e r f c(+) \\
& \left.\quad+k^{2} c K\left(k^{2} t-\frac{|z||k|-2}{2 c K}\right) \mathrm{e}^{-|k||z|} e r f c(-)\right\} . \\
& \frac{\partial^{2} \tilde{S}}{\partial z^{2}}(k, z, t)=-\frac{i k|k|}{2} \tilde{f}(k) \mathrm{e}^{-|k||z|} \mathcal{H}(t) .
\end{aligned}
$$

They indeed fulfill the jump conditions.

\subsubsection{Other inverse Laplace}

The shear stress in Fourier transforms becomes:

$$
\begin{aligned}
\tilde{\sigma}_{x z}= & 2 \mu i k\left\{\frac{\partial \tilde{E}}{\partial z}-z \frac{\partial \tilde{S}}{\partial z}\right\} . \\
= & 2 \mu\left\{\left[\frac{-\left(|k|-|z| k^{2}\right) \tilde{f}(k)}{2}+\frac{|z||k|-1}{2|k| c K} \tilde{g}(k)\right] \mathrm{e}^{-|k||z|} \mathcal{H}(t)\right. \\
& +i k \tilde{g}(k)\left(\frac{t|k|}{2}+\frac{|z||k|+1}{4|k| c K}\right) \mathrm{e}^{|k||z|} \operatorname{erf} c(+) \\
& +i k \tilde{g}(k)\left(\frac{t|k|}{2}-\frac{|z||k|-1}{4|k| c K}\right) \mathrm{e}^{-|k||z|} \operatorname{erf} c(-) \\
& \left.-i k \frac{\tilde{g}(k) t}{\sqrt{\pi c K t}} \mathrm{e}^{-\left(k^{2} c K t+z^{2} /(4 c K t)\right)}\right\} \\
= & -\mu^{2} \tilde{f}(k)|k| \\
c & {\left[-\left(1-\frac{c}{\mu}\right)(1-|z||k|)\right] \mathrm{e}^{-|k||z|} \mathcal{H}(t) } \\
& +c K\left(k^{2} t+\frac{|z||k|+1}{2 c K}\right) \mathrm{e}^{|k||z|} \operatorname{erf} c(+) \\
& +c K\left(k^{2} t-\frac{|z||k|-1}{2 c K}\right) \mathrm{e}^{-|k||z|} \operatorname{erfc}(-) \\
& \left.-2|k| \frac{\sqrt{c K t}}{\sqrt{\pi}} \mathrm{e}^{-\left(k^{2} c K t+z^{2} /(4 c K t)\right)}\right\} \\
&
\end{aligned}
$$

The chemical potential is calculated as

$$
\begin{aligned}
\tilde{\mu} f= & c\left(\frac{\partial^{2}}{\partial z^{2}}-k^{2}\right) \tilde{E}-2 \mu \frac{\partial \tilde{S}}{\partial z}-R T \Gamma C_{0} 2 \pi \delta(k) . \\
= & \frac{\operatorname{sign}(z)}{K} \tilde{g}(k) \mathrm{e}^{-|k||z|} \mathcal{H}(t) \\
& +\frac{\tilde{g}(k) \operatorname{sign}(z)}{2 K}\left(\mathrm{e}^{|k||z|} \operatorname{erfc}(+)+\mathrm{e}^{-|k||z|} \operatorname{erf} c(-)\right) \\
& -i k \mu \tilde{f}(k) \mathrm{e}^{-|k||z|}-R T \Gamma C_{0} 2 \pi \delta(k), \\
= & \frac{i \mu k \tilde{f}(k) \operatorname{sign}(z)}{2}\left\{\mathrm{e}^{|k||z|} \operatorname{erfc}(+)-\mathrm{e}^{-|k||z|} \operatorname{erfc}(-)\right\} \\
& -R T \Gamma C_{0} 2 \pi \delta(k)
\end{aligned}
$$

\subsection{Inverse Fourier transform}

The shear fault is caused by a dislocation. Then, the slip function becomes:

$f(x)=d(\mathcal{H}(L+x)-\mathcal{H}(L-x))$.

The Fourier transformed is

$\tilde{f}(k)=\int_{-L}^{L} \mathrm{de}^{-i k x} \mathrm{~d} x=\frac{2 \mathrm{~d} \sin (L k)}{k}$.

The function $\tilde{g}(k)$ becomes in spatial domain:

$$
\begin{aligned}
g(x) & =\mathcal{F}^{-1}(i k \mu \tilde{f}(k) K) \\
& =-\mu K \frac{\partial f(x)}{\partial x} \\
& =-\mu \mathrm{d} K(\delta(L+x)-\delta(L-x))
\end{aligned}
$$

The solution at the crack surface is considered, namely at $z=0$. Then the shear stress is given by

$$
\begin{aligned}
\tilde{\sigma}_{x z}(k, 0, t) & =-2 \mu\left[\frac{|k| \tilde{f}(k)}{2}+\frac{i k}{2|k| c K} \tilde{g}(k)\right] \mathcal{H}(t) \\
& +2 \mu \tilde{g}(k)\left\{\left(i t k|k|+\frac{i k}{2|k| c K}\right) \operatorname{erf} c(|k| \sqrt{c K t})\right. \\
& \left.-\frac{i k t}{\sqrt{\pi c K t}} \mathrm{e}^{-k^{2} c K t}\right\} \\
= & -\mu^{2} \tilde{f}(k)|k| \\
c & {\left[-\left(1-\frac{c}{\mu}\right)\right] \mathcal{H}(t) } \\
& +\left(2 k^{2} c K t+1\right) \operatorname{erf}(|k| \sqrt{c K t}) \\
& \left.-2 \frac{\sqrt{k^{2} c K t}}{\sqrt{\pi}} \mathrm{e}^{-\left(k^{2} c K t\right)}\right\}
\end{aligned}
$$

Abramowitz and Stegun (1970, paragraph 7.2) deals with repeated integrals of the complementary error function $i^{n} \operatorname{erfc}(\lambda)$ : 
$i^{n} \operatorname{erfc}(\lambda)=\int_{\lambda}^{\infty} i^{n-1} \operatorname{erfc}(l) \mathrm{d} l$.

with

$$
\begin{aligned}
i^{n} \operatorname{erfc}(\lambda) & =\frac{2}{\sqrt{\pi}} \int_{\lambda}^{\infty} \frac{(l-\lambda)^{n}}{n !} \mathrm{e}^{-l^{2}} \mathrm{~d} l, \\
& =-\frac{\lambda}{n} i^{n-1} \operatorname{erfc}(\lambda)+\frac{1}{2 n} i^{n-2} \operatorname{erfc}(\lambda)
\end{aligned}
$$

$i^{0} \operatorname{erfc}(\lambda)=\operatorname{erfc}(\lambda)$,

$i^{-1} \operatorname{erfc}(\lambda)=\frac{2}{\sqrt{\pi}} \mathrm{e}^{-\lambda^{2}}$

We can derive that

$i^{1} \operatorname{erfc}(\lambda)=-\lambda \operatorname{erfc}(\lambda)+\frac{1}{\sqrt{\pi}} \mathrm{e}^{-\lambda^{2}}$.

$i^{2} \operatorname{erfc}(\lambda)=\frac{1}{4}\left\{\left(1+2 \lambda^{2}\right) \operatorname{erfc}(\lambda)-2 \frac{\lambda}{\sqrt{\pi}} \mathrm{e}^{-\lambda^{2}}\right\}$

With $\lambda=\sqrt{k^{2} c K t}$ and a factor $\frac{2 i k}{c K|k|}$ we have found for (101)

$$
\begin{aligned}
\tilde{\sigma}_{x z}(k, 0, t)= & \frac{-\mu^{2}|k| \tilde{f}(k)}{c}\left\{-\left(1-\frac{c}{\mu}\right) \mathcal{H}(t)\right. \\
& \left.+4 i^{2} \operatorname{erfc}\left(\sqrt{k^{2} c K t}\right)\right\} .
\end{aligned}
$$

For calculating the inverse Fourier, the first and second terms are calculated separately. Since $\tilde{f}(k)$ is symmetric and $|k|$ is symmetric, the first term is symmetric. For the inverse Fourier transformation this means that only the real part, i.e. symmetric part, of $\mathrm{e}^{i k x}=\cos (k x)+i \sin (k x)$ contributes:

$$
\begin{aligned}
& \mathcal{F}^{-1}(|k| \tilde{f}(k))=\frac{2 d}{\pi} \int_{0}^{\infty} \sin (k L) \cos (k x) \mathrm{d} k \\
& =\left.\frac{2 d}{\pi} \frac{(L-x) \cos ((L+x) k)+(L+x) \cos ((L-x) k)}{2\left(x^{2}-L^{2}\right)}\right|_{0} ^{\infty} \\
& =\frac{2 d}{\pi} \frac{L}{\left(L^{2}-x^{2}\right)}
\end{aligned}
$$

The error function $i^{2} \operatorname{erf} c\left(\sqrt{k^{2} c K t}\right)$ is symmetric in $k$. This means that the second part of the shear stress is symmetric in $k$ too. The real part of $\mathrm{e}^{i k x}$ only contributes. This way the inverse Fourier reduces to

$$
\begin{aligned}
\mathcal{F}^{-1} & \left(|k| \tilde{f}(k) i^{2} \operatorname{erfc}\left(\sqrt{k^{2} c K t}\right)\right) \\
& =\int_{-\infty}^{\infty} \tilde{f}(k)|k| i^{2} \operatorname{erfc}\left(\sqrt{k^{2} c K t}\right) \mathrm{e}^{i k} \mathrm{~d} k \\
& =\frac{2 d}{\pi} \int_{0}^{\infty} i^{2} \operatorname{erfc}\left(\sqrt{k^{2} c K t}\right) \sin (k L) \cos (k x) \mathrm{d} k \\
& =\frac{2 d}{\pi} \operatorname{Int}_{i^{2}} \operatorname{erfc} .
\end{aligned}
$$

The integral $\operatorname{Int}_{i^{2}} \operatorname{erfc}=\int_{0}^{\infty} i^{2} \operatorname{erf} c\left(\sqrt{k^{2} c K t}\right) \sin (k L)$ $\cos (k x) \mathrm{d} k$ is introduced. By partial integration we find that the next integral can be derived. The property $\lim _{x \rightarrow \infty} i^{n}$ $\operatorname{erfc}(x)=0$ is used.

$$
\begin{aligned}
& \operatorname{Int}_{i^{2} e r f c} \\
& =\int_{0}^{\infty} i^{2} \operatorname{erfc}\left(\sqrt{k^{2} c K t}\right) \sin (k L) \cos (k x) \mathrm{d} k \\
& =\left[\frac{1}{x} i^{2} \operatorname{erfc}\left(\sqrt{k^{2} c K t}\right) \sin (k L) \sin (k x)\right]_{0}^{\infty} \\
& -\int_{0}^{\infty}\left\{\frac{-\sqrt{c K t}}{x} i^{1} \operatorname{erfc}\left(\sqrt{k^{2} c K t}\right) \sin (k L)\right. \\
& \left.+\frac{L}{x} i^{2} \operatorname{erfc}\left(\sqrt{k^{2} c K t}\right) \cos (k L)\right\} \sin (k x) \mathrm{d} k, \\
& {\left[\left\{\frac{-\sqrt{c K t}}{x^{2}} i^{1} \operatorname{erfc}\left(\sqrt{k^{2} c K t}\right) \sin (k L)\right.\right.} \\
& \left.\left.+\frac{L}{x^{2}} i^{2} \operatorname{erfc}\left(\sqrt{k^{2} c K t}\right) \cos (k L)\right\} \cos (k x)\right]_{0}^{\infty} \\
& -\int_{0}^{\infty}\left\{\frac{c K t}{x^{2}} \operatorname{erfc}\left(\sqrt{k^{2} c K t}\right) \sin (k L)\right. \\
& \left.-2 \frac{L \sqrt{c K t}}{x^{2}} i^{1} \operatorname{erfc}\left(\sqrt{k^{2} c K t}\right) \cos (k L)\right\} \cos (k x) \mathrm{d} k \\
& +\frac{L^{2}}{x^{2}} \operatorname{Int} t^{2} e r f c, \\
& -\frac{L}{x^{2}} i^{2} \operatorname{erf} c(0)+\frac{L^{2}}{x^{2}} \operatorname{Int}_{i^{2} e r f c} \\
& -\frac{c K t}{x^{2}} \int_{0}^{\infty} \operatorname{erfc}\left(\sqrt{k^{2} c K t}\right) \sin (k L) \cos (k x) \mathrm{d} k \\
& +2 \frac{L \sqrt{c K t}}{x^{2}} \int_{0}^{\infty} i^{1} \operatorname{erf} c\left(\sqrt{k^{2} c K t}\right) \cos (k L) \cos (k x) \mathrm{d} k,
\end{aligned}
$$




$$
\begin{aligned}
= & \frac{1}{L^{2}-x^{2}}\left\{+L i^{2} \operatorname{erf} c(0)+c K t \operatorname{Int}_{\operatorname{erf} c}\right. \\
& \left.-2 L \sqrt{c K t} \operatorname{Int}_{i^{1}} \operatorname{erfc}\right\} .
\end{aligned}
$$

We denote $\operatorname{Int}_{i^{1} \operatorname{erf} c}=\int_{0}^{\infty} \operatorname{erf} c\left(\sqrt{k^{2} c K t}\right) \cos (k L) \cos (k x) \mathrm{d} k$ :

$$
\begin{aligned}
& \operatorname{Int}_{i}{ }^{1} \operatorname{erfc} \\
& =\int_{0}^{\infty} \operatorname{erfc}\left(\sqrt{k^{2} c K t}\right) \cos (k L) \cos (k x) \mathrm{d} k \\
& =0-\frac{1}{x} \int_{0}^{\infty}\left\{-\sqrt{c K t} \operatorname{erf} c\left(\sqrt{k^{2} c K t}\right) \cos (k L)\right. \\
& \left.-L i^{1} \operatorname{erf} c\left(\sqrt{k^{2} c K t}\right) \sin (k L)\right\} \sin (k x) \mathrm{d} k \\
& =\frac{\sqrt{c K t}}{x} \int_{0}^{\infty} \operatorname{erfc}\left(\sqrt{k^{2} c K t}\right) \cos (k L) \sin (k x) \mathrm{d} k \\
& -\frac{L}{x^{2}} \int_{0}^{\infty} i^{1} \operatorname{erf} c\left(\sqrt{k^{2} c K t}\right) \sin (k L) d \cos (k x), \\
& =\frac{\sqrt{c K t}}{x} \int_{0}^{\infty} \operatorname{erfc}\left(\sqrt{k^{2} c K t}\right) \cos (k L) \sin (k x) \mathrm{d} k-0 \\
& +\frac{L}{x^{2}} \int_{0}^{\infty}\left\{-\sqrt{c K t} \operatorname{erfc}\left(\sqrt{k^{2} c K t}\right) \sin (k L)\right. \\
& \left.+L i^{1} \operatorname{erf} c\left(\sqrt{k^{2} c K t}\right) \cos (k L)\right\} \cos (k x) \mathrm{d} k \\
& =\frac{\sqrt{c K t}}{x} \int_{0}^{\infty} \operatorname{erfc}\left(\sqrt{k^{2} c K t}\right)\{\cos (k L) \sin (k x) \\
& \left.-\frac{L}{x} \sin (k L) \cos (k x)\right\} \mathrm{d} k+\frac{L^{2}}{x^{2}} \operatorname{Int}_{i^{1} e r f c}, \\
& =-\frac{x \sqrt{c K t}}{L^{2}-x^{2}} \int_{0}^{\infty} \operatorname{erfc}\left(\sqrt{k^{2} c K t}\right)\{\cos (k L) \sin (k x) \\
& \left.-\frac{L}{x} \sin (k L) \cos (k x)\right\} \mathrm{d} k \text {. }
\end{aligned}
$$

The integral left is similar to Int $t_{e r f}$, but with $x$ and $L$ the other way around. We denote

$$
\begin{aligned}
& \text { Int }_{\text {erfc }}=\int_{0}^{\infty} \operatorname{erfc}\left(\sqrt{k^{2} c K t}\right) \sin (k L) \cos (k x) \mathrm{d} k: \\
& \text { Int }_{\text {erf } c} \\
& =\int_{0}^{\infty} \operatorname{erfc}\left(\sqrt{k^{2} c K t}\right) \sin (k L) \cos (k x) \mathrm{d} k \\
& =\left[\frac{1}{x} \operatorname{erfc}\left(\sqrt{k^{2} c K t}\right) \sin (k L) \sin (k x)\right]_{0}^{\infty}
\end{aligned}
$$

$$
\begin{aligned}
& -\int_{0}^{\infty} \frac{1}{x}\left\{-\frac{2 \sqrt{c K t}}{\sqrt{\pi}} \mathrm{e}^{-k^{2} c K t} \sin (k L)\right. \\
& \left.+\operatorname{Lerfc}\left(\sqrt{k^{2} c K t}\right) \cos (k L)\right\} \sin (k x) \mathrm{d} k
\end{aligned}
$$$$
=0+\int_{0}^{\infty} \frac{1}{x} \frac{2 \sqrt{c K t}}{\sqrt{\pi}} \mathrm{e}^{-k^{2} c K t} \sin (k L) \sin (k x) \mathrm{d} k
$$$$
+\left[\frac{L}{x^{2}} \operatorname{erfc}\left(\sqrt{k^{2} c K t}\right) \cos (k L) \cos (k x)\right]_{0}^{\infty}
$$$$
-\frac{L}{x^{2}} \int_{0}^{\infty} \frac{-2 \sqrt{c K t}}{\sqrt{\pi}} \mathrm{e}^{-k^{2} c K t} \cos (k L) \cos (k x) \mathrm{d} k+\frac{L^{2}}{x^{2}} \operatorname{Int} t_{e r f}
$$$$
=-\frac{\operatorname{Lerfc}(0)}{x^{2}}+\int_{0}^{\infty} \frac{1}{x} \frac{2 \sqrt{c K t}}{\sqrt{\pi}} \mathrm{e}^{-k^{2} c K t}\{\sin (k L) \sin (k x)
$$$$
\left.+\frac{L}{x} \cos (k L) \cos (k x)\right\} \mathrm{d} k+\frac{L^{2}}{x^{2}} \operatorname{Int} t_{e r f c}
$$$$
=\frac{1}{L^{2}-x^{2}}\left\{\operatorname{Lerfc}(0)-\frac{2 x \sqrt{c K t}}{\sqrt{\pi}} \operatorname{Int}_{\exp }\right\}
$$

The last unknown integral is $I n t_{\text {exp }}$ :

$$
\begin{aligned}
& \text { Int } t_{\text {exp }} \\
& =\int_{0}^{\infty} \mathrm{e}^{-k^{2} c K t}\left\{\sin (k L) \sin (k x)+\frac{L}{x} \cos (k L) \cos (k x)\right\} \mathrm{d} k \\
& =\int_{0}^{\infty} \mathrm{e}^{-k^{2} c K t}\left\{\frac{(L-x)}{2 x} \cos ((L+x) k)\right. \\
& \left.+\frac{(L+x)}{2 x} \cos ((L-x) k)\right\} \mathrm{d} k, \\
& =\frac{(L-x)}{4 x} \int_{0}^{\infty}\left\{\mathrm{e}^{-k^{2} c K t+i(L+x) k}+\mathrm{e}^{-k^{2} c K t-i(L+x) k}\right\} \mathrm{d} k \\
& +\frac{(L+x)}{4 x} \int_{0}^{\infty}\left\{\mathrm{e}^{-k^{2} c K t+i(L-x) k}+\mathrm{e}^{-k^{2} c K t-i(L-x) k}\right\} \mathrm{d} k, \\
& =\frac{(L-x)}{4 x} \int_{0}^{\infty} \mathrm{e}^{-\frac{(L+x)^{2}}{4 c K t}}\left\{\mathrm{e}^{-\left(k \sqrt{c K t}+i \frac{(L+x)}{2 \sqrt{c K t}}\right)^{2}}\right. \\
& \left.+\mathrm{e}^{-\left(k \sqrt{c K t}-i \frac{(L+x)}{2 \sqrt{c K t}}\right)^{2}}\right\} \mathrm{d} k \\
& +\frac{(L+x)}{4 x} \int_{0}^{\infty} \mathrm{e}^{-\frac{(L-x)^{2}}{4 c K t}}\left\{\mathrm{e}^{-\left(k \sqrt{c K t}+i \frac{(L-x)}{2 \sqrt{c K t}}\right)^{2}}\right. \\
& \left.+\mathrm{e}^{-\left(k \sqrt{c K t}-i \frac{(L-x)}{2 \sqrt{c K t}}\right)^{2}} \mathrm{e}^{-\left(k \sqrt{c K t}+i \frac{(L+x)}{2 \sqrt{c K t}}\right)^{2}}\right\} \mathrm{d} k, \\
& =-\frac{(L+x)}{4 x} \mathrm{e}^{\frac{(L-x)^{2}}{4 c K t}} \frac{\sqrt{\pi}}{2 \sqrt{c K t}}\left\{\operatorname{erfc}\left(-i \frac{(L+x)}{2 \sqrt{c K t}}\right)\right.
\end{aligned}
$$




$$
\begin{aligned}
& \left.+\operatorname{erfc}\left(i \frac{(L+x)}{2 \sqrt{c K t}}\right)\right\} \\
& +\frac{(L+x)}{4 x} \mathrm{e}^{-\frac{(L-x)^{2}}{4 c K t}} \frac{\sqrt{\pi}}{2 \sqrt{c K t}}\left\{\operatorname{erfc}\left(-i \frac{(L-x)}{2 \sqrt{c K t}}\right)\right. \\
& \left.+\operatorname{erfc}\left(i \frac{(L-x)}{2 \sqrt{c K t}}\right)\right\} \\
& =\frac{(L-x)}{4 x} \frac{\sqrt{\pi}}{\sqrt{c K t}} \mathrm{e}^{-\frac{(L+x)^{2}}{4 c K t}}+\frac{(L+x)}{4 x} \frac{\sqrt{\pi}}{\sqrt{c K t}} \mathrm{e}^{-\frac{(L-x)^{2}}{4 c K t}} .
\end{aligned}
$$

Then

$$
\begin{aligned}
& \text { Interf } c \\
& \begin{aligned}
= & \frac{\operatorname{Lerf} c(0)}{L^{2}-x^{2}}-\frac{1}{2(L+x)} \mathrm{e}^{-\frac{(L+x)^{2}}{4 c K t}} \\
& -\frac{1}{2(L-x)} \mathrm{e}^{-\frac{(L-x)^{2}}{4 c K t}} \\
\text { Int }_{i^{1}} \operatorname{erf} c & \\
= & \frac{\sqrt{c K t}}{L^{2}-x^{2}}\left\{\frac{L^{2}+x^{2}}{L^{2}-x^{2}} \operatorname{erfc}(0)-\frac{(L-x)}{2(L+x)} \mathrm{e}^{-\frac{(L+x)^{2}}{4 c K t}}\right. \\
& \left.-\frac{(L+x)}{2(L-x)} \mathrm{e}^{-\frac{(L-x)^{2}}{4 c K t}}\right\} \\
= & \sqrt{c K t}\left\{\frac{L^{2}+x^{2}}{\left(L^{2}-x^{2}\right)^{2}} \operatorname{erfc}(0)-\frac{1}{2(L+x)^{2}} \mathrm{e}^{-\frac{(L+x)^{2}}{4 c K t}}\right. \\
& \left.-\frac{1}{2(L-x)^{2}} \mathrm{e}^{-\frac{(L-x)^{2}}{4 c K t}}\right\}
\end{aligned}
\end{aligned}
$$

All the separate integrals have been determined and we find for $\operatorname{Int}_{i^{2}} \operatorname{erfc}$ the next solution.

$$
\begin{aligned}
\operatorname{Int}_{i^{2} e r f c}= & \frac{1}{L^{2}-x^{2}}\left\{L i^{2} \operatorname{erf} c(0)+c K \text { Int }_{\text {erf } c}\right. \\
& \left.-2 L \sqrt{c K t} \operatorname{Int}_{i^{1} e r f c}\right\} \\
= & \frac{1}{\left(L^{2}-x^{2}\right)}\left\{L i^{2} \operatorname{erf} c(0)\right. \\
& -c K t \frac{3 L x^{2}+L^{3}}{\left(L^{2}-x^{2}\right)^{2}} \operatorname{erf} c(0) \\
& +c K t\left(\frac{(L-x)}{2(L+x)^{2}} \mathrm{e}^{-\frac{(L+x)^{2}}{4 c K t}}\right. \\
& \left.\left.+\frac{(L+x)}{2(L-x)^{2}} \mathrm{e}^{-\frac{(L-x)^{2}}{4 c K t}}\right)\right\} .
\end{aligned}
$$

Furthermore, for the repetitive error function holds:

$$
\begin{aligned}
& i^{n} \operatorname{erfc}(\infty)=0 \\
& i^{n} \operatorname{erfc}(0)=\left[2^{n} \Gamma(n / 2+1)\right]^{-1} \\
& \Gamma(n)=(n-1) !, n \in \mathcal{N}^{+} .
\end{aligned}
$$

The next holds:

$$
\frac{(L+x)}{2(L-x)^{2}}+\frac{(L-x)}{2(L+x)^{2}}=\frac{3 L x^{2}+L^{3}}{\left(L^{2}-x^{2}\right)^{2}}
$$

We have found for $\operatorname{Int}_{i^{2}} \operatorname{erfc}$

$$
\begin{aligned}
\operatorname{Int}_{i^{2} e r f c}= & \frac{1}{L^{2}-x^{2}}\left\{\frac{L}{4}-c K t\left(\frac{(L-x)}{2(L+x)^{2}}\left(1-\mathrm{e}^{-\frac{(L+x)^{2}}{4 c K t}}\right)\right.\right. \\
& \left.\left.+\frac{(L+x)}{2(L-x)^{2}}\left(1-\mathrm{e}^{-\frac{(L-x)^{2}}{4 c K t}}\right)\right)\right\}
\end{aligned}
$$

Then, the shear stress is given by Eq. (105)

$$
\begin{aligned}
\sigma_{x z}(x, 0, t)= & \mathcal{F}^{-1}\left(\frac { - \mu ^ { 2 } | k | \tilde { f } ( k ) } { c } \left\{-\left(1-\frac{c}{\mu}\right) \mathcal{H}(t)\right.\right. \\
& \left.\left.+4 i^{2} \operatorname{erf}\left(\sqrt{k^{2} c K t}\right)\right\}\right) \\
= & \frac{-\mu^{2} \mathcal{H}(t)}{c}\left\{-\left(1-\frac{\mu}{c}\right) \mathcal{F}^{-1}(|k| \tilde{f}(k))\right. \\
& \left.+4 \mathcal{F}^{-1}\left(i^{2} \operatorname{erf} c\left(\sqrt{k^{2} c K t}|k| \tilde{f}(k)\right)\right)\right\} \\
& =\frac{-2 \mu \mathcal{H}(t) d}{\pi}\left\{\left(1-\frac{\mu}{c}\right) \frac{L}{L^{2}-x^{2}}\right. \\
& \left.+4 \frac{\mu}{c} \operatorname{Int}_{i^{2}} \operatorname{erf} c\right\},
\end{aligned}
$$

which leads to

$\sigma_{x z}(x, 0, t)=\frac{-2 \mu \mathcal{H}(t) d}{\pi\left(L^{2}-x^{2}\right)}\{L-\mu K F(x, t)$.

with

$$
\begin{aligned}
F(x, t)= & 2 t \frac{(L-x)}{(L+x)^{2}}\left(1-\mathrm{e}^{-\frac{(L+x)^{2}}{4 c K t}}\right) \\
& +\frac{(L+x)}{(L-x)^{2}}\left(1-\mathrm{e}^{-\frac{(L-x)^{2}}{4 c K t}}\right)
\end{aligned}
$$

\subsection{Chemical potential and flow}

The chemical potential does not only satisfies Eq. (9), but it also satisfies Eq. (28), with $S$ time independent. Assuming separation of polar variables $r$ and $\theta$ about the crack tip, we solve the $r$-dependent part of the solution using Hankel transformation (Booker 1974; Nur and Booker 1972). The result is that the chemical potential follows

$\mu^{\mathrm{f}}=\left(1-\mathrm{e}^{-r^{2} / 4 c K t}\right) \mu_{0}^{\mathrm{f}} \quad \mu_{0}^{\mathrm{f}}=A L \frac{\sin (\theta)}{r}$

with local polar coordinate $r$ defined as the radius at one of the two crack-tips and $\theta$ the angle with $x$-axis. The parameter $A$ is a measure for the offset: $A=\frac{2 \mu d b}{2 \pi L}$. In $x, z$-plane, this becomes

$$
\begin{aligned}
\mu^{\mathrm{f}}= & \frac{2 \mu d}{L} \frac{z}{2 \pi L}\left[\frac{L^{2}}{(x-L)^{2}+z^{2}}-\frac{L^{2}}{(x+L)^{2}+z^{2}}\right] \\
& -\frac{2 \mu d}{L} \frac{z}{2 \pi L} \mathrm{e}^{-z^{2} / 4 c K t} \\
& \times\left[\frac{L^{2} \mathrm{e}^{-(x-L)^{2} / 4 c K t}}{(x-L)^{2}+z^{2}}-\frac{L^{2} \mathrm{e}^{-(x+L)^{2} / 4 c K t}}{(x+L)^{2}+z^{2}}\right]
\end{aligned}
$$


and therefore (122) holds.

$$
\begin{aligned}
\mu^{\mathrm{f}=}= & \frac{\mu d z}{\pi}\left[\frac{1}{(x-L)^{2}+z^{2}}-\frac{1}{(x+L)^{2}+z^{2}}\right] \\
& -\frac{\mu d z}{\pi} \mathrm{e}^{-z^{2} / 4 c K t}\left[\frac{\mathrm{e}^{-(x-L)^{2} / 4 c K t}}{(x-L)^{2}+z^{2}}-\frac{\mathrm{e}^{-(x+L)^{2} / 4 c K t}}{(x+L)^{2}+z^{2}}\right]
\end{aligned}
$$

From this, we can derive the flow over the crack (n vector normal to the crack):

$$
\begin{aligned}
\mathbf{q} \cdot \mathbf{n}= & -K \frac{\partial \mu^{\mathrm{f}}}{\partial z} \\
& =-K \frac{\mu d}{\pi}\left[\frac{1}{(x-L)^{2}+z^{2}}-\frac{1}{(x+L)^{2}+z^{2}}\right] \\
& +K \frac{2 \mu d z^{2}}{\pi}\left[\frac{1}{\left((x-L)^{2}+z^{2}\right)^{2}}-\frac{1}{\left((x+L)^{2}+z^{2}\right)^{2}}\right] \\
& +K \frac{\mu d\left(1-2 z^{2} / 4 c K t\right)}{\pi} \mathrm{e}^{-z^{2} / 4 c K t}\left[\frac{\mathrm{e}^{-(x-L)^{2} / 4 c K t}}{(x-L)^{2}+z^{2}}\right. \\
& \left.-\frac{\mathrm{e}^{-(x+L)^{2} / 4 c K t}}{(x+L)^{2}+z^{2}}\right] \\
& -K \frac{2 \mu d z^{2}}{\pi} \mathrm{e}^{-z^{2} / 4 c K t}\left[\frac{\mathrm{e}^{-(x-L)^{2} / 4 c K t}}{\left((x-L)^{2}+z^{2}\right)^{2}}\right. \\
& \left.-\frac{\mathrm{e}^{-(x+L)^{2} / 4 c K t}}{\left((x+L)^{2}+z^{2}\right)^{2}}\right]
\end{aligned}
$$

and the flow along the crack ( $\mathbf{t}$ vector in direction of crack-tip $x=+L)$ :

$$
\begin{aligned}
\mathbf{q} \cdot \mathbf{t}= & -K \frac{\partial \mu^{\mathrm{f}}}{\partial x} \\
= & \frac{\mu d z}{\pi}\left[\frac{-2(x-L)}{(x-L)^{2}+z^{2}}\right. \\
& \left.-\frac{-2(x+L)}{(x+L)^{2}+z^{2}}\right] \\
& -\frac{\mu d z}{\pi} \mathrm{e}^{-z^{2} / 4 c K t}\left[\frac{\frac{-2(x-L)}{4 c K t} \mathrm{e}^{-(x-L)^{2} / 4 c K t}}{(x-L)^{2}+z^{2}}\right. \\
& \left.-\frac{\frac{-2(x+L)}{4 c K t} \mathrm{e}^{-(x+L)^{2} / 4 c K t}}{(x+L)^{2}+z^{2}}\right] \\
& -\frac{\mu d z}{\pi} \mathrm{e}^{-z^{2} / 4 c K t}\left[\frac{-2(x-L) \mathrm{e}^{-(x-L)^{2} / 4 c K t}}{\left((x-L)^{2}+z^{2}\right)^{2}}\right. \\
& \left.-\frac{-2(x+L) \mathrm{e}^{-(x+L)^{2} / 4 c K t}}{\left((x+L)^{2}+z^{2}\right)^{2}}\right]
\end{aligned}
$$

\section{References}

Abramowitz M, Stegun IA (1970) Handbook of mathematical functions. Dover, New York
Armero F, Callari C (1999) An analysis of strong discontinuities in a saturated poro-plastic solid. Int $\mathrm{J}$ Numer Methods Eng 46(10):1673-1698

Atkinson C, Craster RV (1991) Plane-strain fracture in poroelastic media. Proc R Soc Lond Ser A Math Phys Eng Sci 434(1892):605633

Biot MA (1941) General theory of three-dimensional consolidation. J Appl Phys 12(2):155-164

Biot MA (1956) General solutions of the equations of elasticity and consolidation for a porous material. J Appl Mech 78:91-96

Booker JR (1974) Time-dependent strain following faulting of a porous-medium. J Geophys Res 79(14):2037-2044

Chen Y, Chen X, Hisada T (2006) Non-linear finite element analysis of mechanical electrochemical phenomena in hydrated soft tissues based on triphasic theory. Int J Numer Methods Eng 65:147-173

Detournay E, Cheng AHD (1993) Comprehensive rock engineering: principles, practice and projects, vol II. Pergamon

Frijns AJH, Huyghe JM, Janssen JD (1997) A validation of the quadriphasic mixture theory for intervertebral disc tissue. Int J Eng Sci 35(15):1419-1429

Gu W, Lai W, Mow V (1998) A mixture theory for charged-hydrated soft tissues containing multi-electrolytes: passive transport and swelling behaviors. J Biomech Eng 120:169-180

Huyghe JM, Janssen JD (1997) Quadriphasic mechanics of swelling incompressible porous media. Int J Eng Sci 35(8):793-802

Huyghe JM, Janssen JD (1999) Thermo-chemo-electro-mechanical formulation of saturated charged porous solids. Transp Porous Media 34(1-3):129-141

Iatridis JC, Laible JP, Krag MH (2003) Influence of fixed charge density magnitude and distribution on the intervertebral disc: applications of a poroelastic and chemical electric (peace) model. J Biomech Eng 125:12-24

Lai WM, Hou JS, Mow VC (1991) A triphasic theory for the swelling and deformation behaviors of articular-cartilage. J Biomech Eng Trans Asme 113(3):245-258

Lanir Y (1987) Biorheology and fluid flux in swelling tissues. 1. Bicomponent theory for small deformations, including concentration effects. Biorheology 24(2):173-187

Larsson J, Larsson R (2000) Localization analysis of a fluid-saturated elastoplastic porous medium using regularized discontinuities. Mech Cohes Frict Mater 5(7):565-582

Loret B, Radi E (2001) The effects of inertia on crack growth in poroelastic fluid-saturated media. J Mech Phys Solids 49(5):995-1020

Maroudas A, Wachtel E, Grushko G, Katz EP, Weinberg P (1991) The effect of osmotic and mechanical pressures on water partitioning in articular cartilage. Biochim et Biophys Acta 1073:285-294

McNamee J, Gibson RE (1960) Displacements functions and linear transforms applied to diffusion through porous elastic media. Q J Mech Appl Math 13(1):98-111

Meerveld JV, Molenaar MM, Huyghe JM, Baaijens FPT (2003) Analytical solution of compression, free swelling and electrical loading of saturated charged porous media. Transp Porous Media 50(1-2):111-126

Nur A, Booker JR (1972) Aftershocks caused by pore fluid-flow. Science 175(4024):885-887

Rethore J, de Borst R, Abellan MA (2007) A two-scale approach for fluid flow in fractured porous media. Int J Numer Methods Eng 71(7):780-800

Rice JR, Cleary MP (1976) Some basic stress diffusion solutions for fluid-saturated elastic porous media with compressible constituents. Rev Geophys Space Phys 14(2):227-241

Schrefler BA, Secchio S, Simoni L (2004) Numerical procedure for discrete fracture propagation in porous materials, pp 220-229

Schroeder Y, Wilson W, Huyghe JM, Baaijens FP (2006) Osmoviscoelastic finite element model of the intervertebral disc. Eur Spine J 15(Suppl 3):S361-S371 
Urban JP, Roberts S (2003) Degeneration of the intervertebral disc. Arthritis Res Ther 5(3):120-130

van Loon R, Huyghe JM, Wijlaars MW, Baaijens FPT (2003) 3d fe implementation of an incompressible quadriphasic mixture model. Int J Numer Methods Eng 57(9):1243-1258

Verruijt A (1971) Displacement functions in theory consolidation or in thermoelasticity. Zeitschrift fur Angewandte Mathematik und Physik 22(5):891-898

Wilson W, van Donkelaar CC, van Rietbergen B, Ito K, Huiskes $R$ (2004) Stresses in the local collagen network of articular cartilage: a poroviscoelastic fibril-reinforced finite element study. J Biomech 37(3):357-366
Wilson W, van Donkelaar CC, Huyghe JM (2005) A comparison between mechano-electrochemical and biphasic swelling theories for soft hydrated tissues. J Biomech Eng Trans Asme 127(1):158165

Wilson W, van Donkelaar CC, van Rietbergen B, Huiskes R (2005) A fibril-reinforced poroviscoelastic swelling model for articular cartilage. J Biomech. 38(6):1195-1204

Wognum S, Huyghe JM, Baaijens FPT (2006) Influence of osmotic pressure changes on the opening of existing cracks in 2 intervertebral disc models. Spine 31(16):1783-1788 